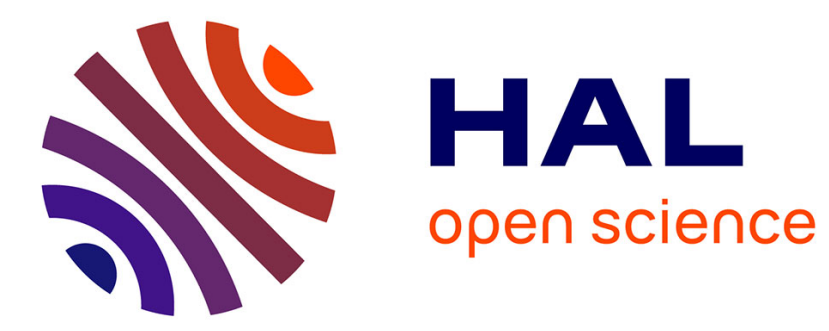

\title{
LCC-Demons: a robust and accurate symmetric diffeomorphic registration algorithm
}

Marco Lorenzi, Nicholas Ayache, Giovanni B. Frisoni, Xavier Pennec

\section{To cite this version:}

Marco Lorenzi, Nicholas Ayache, Giovanni B. Frisoni, Xavier Pennec. LCC-Demons: a robust and accurate symmetric diffeomorphic registration algorithm. NeuroImage, 2013, 81 (1), pp.470-483. 10.1016/j.neuroimage.2013.04.114 . hal-00819895v2

\section{HAL Id: hal-00819895 \\ https://hal.inria.fr/hal-00819895v2}

Submitted on 20 Jan 2014

HAL is a multi-disciplinary open access archive for the deposit and dissemination of scientific research documents, whether they are published or not. The documents may come from teaching and research institutions in France or abroad, or from public or private research centers.
L'archive ouverte pluridisciplinaire HAL, est destinée au dépôt et à la diffusion de documents scientifiques de niveau recherche, publiés ou non, émanant des établissements d'enseignement et de recherche français ou étrangers, des laboratoires publics ou privés. 


\title{
LCC-Demons: a robust and accurate symmetric diffeomorphic registration algorithm
}

\author{
M. Lorenzi ${ }^{\mathrm{a}, \mathrm{b}, *}$, N. Ayache ${ }^{\mathrm{a}}$, G.B. Frisoni ${ }^{\mathrm{b}}$, X. Pennec $^{\mathrm{a}}$, for the Alzheimer's \\ Disease Neuroimaging Initiative (ADNI) कै \\ ${ }^{a}$ Asclepios Research Project, INRIA Sophia Antipolis, 2004 route des Lucioles BP 93, 06 \\ 902 Sophia Antipolis, France \\ ${ }^{b}$ LENITEM, IRCCS San Giovanni di Dio Fatebenefratelli, via Pilastroni 4, 25125, Brescia, \\ Italy.
}

\begin{abstract}
Non-linear registration is a key instrument for computational anatomy to study the morphology of organs and tissues. However, in order to be an effective instrument for the clinical practice, registration algorithms must be computationally efficient, accurate and most importantly robust to the multiple biases affecting medical images. In this work we propose a fast and robust registration framework based on the log-Demons diffeomorphic registration algorithm. The transformation is parameterized by stationary velocity fields (SVFs), and the similarity metric implements a symmetric local correlation coefficient (LCC). Moreover, we show how the SVF setting provides a stable and consistent numerical scheme for the computation of the Jacobian determinant and the flux of the deformation across the boundaries of a given region. Thus, it provides a robust evaluation of spatial changes. We tested the LCC-Demons in the inter-subject registration setting, by comparing with state-of-the-art registration algorithms on public available datasets, and in the intra-subject longitudinal registration problem, for the statistically powered measurements of the longitudinal atrophy in Alzheimer's disease. Experimental results show that LCC-Demons is a generic, flexible, efficient and robust algorithm for the accurate non-linear registration of images, which can find several applications in the field of medical imaging. Without any additional optimization, it solves equally well intra \&
\end{abstract}

\footnotetext{
${ }^{\text {in }}$ Data used in preparation of this article were obtained from the Alzheimers Disease Neuroimaging Initiative (ADNI) database (adni.loni.ucla.edu). As such, the investigators within the ADNI contributed to the design and implementation of ADNI and/or provided data but did not participate in analysis or writing of this report. A complete listing of ADNI investigators can be found at: ' 'http://adni.loni.ucla.edu/wp-content/uploads/how_to_apply/ ADNI_Acknowledgement_List.pdf' '

is This work was partially funded by the European Research Council (ERC advanced Grant MedYMA), ANR blanc Karametria and the EU project Care4Me.

* Corresponding author

Email addresses: marco.lorenzi@inria.fr (M. Lorenzi), nicholas.ayache@inria.fr (N. Ayache), gfrisoni@fatebenefratelli.it (G.B. Frisoni), xavier.pennec@inria.fr (X. Pennec)
} 
inter-subject registration problems, and compares favorably to state-of-the-art methods.

Keywords: Non-linear Registration, Longitudinal Atrophy, Alzheimer's Disease, Optimization, Demons

\section{Introduction}

In the recent past, computational anatomy acquired an increasing weight in the analysis of medical data and several methods have been developed to study organs in the cross-sectional and longitudinal settings. The cross-sectional approach evaluates the geometrical differences between subjects and highlights the morphological differences between clinical groups. The longitudinal perspective evaluates the changes in time from serial data of the same subject acting as his own control, and is more useful in detecting the subtle changes related to biological processes.

The key instrument of computational anatomy is non-linear registration, which allows to retrieve morphological differences as deformation fields. A great variety of registration techniques have been proposed in medical imaging, depending on the practical application and the theoretical requirements.

\subsection{Non-linear registration in medical imaging: technical and clinical require-} ments.

Assessing the performance of non-linear registration methods is a quite controversial issue, since there is not a univocal way to define registration accuracy and reliability. We can however define a general set of good properties that a registration method should satisfy for the successful use in research and clinic. For this purpose we identify two main applications of non-linear registration: analysis of correspondences and analysis of deformations.

The former application concerns the comparison of anatomical regions between different subjects. In this case the registration algorithm should provide a good alignment of homologous anatomical structures. This requirement is classically tested in the inter-subject registration setting, by providing overlap and similarity measures with respect to ground truth data, given for example by manual segmentation of selected anatomical regions.

The second application concerns the analysis and quantification of anatomical changes by studying the estimated deformations. A classical example of such an application is the Tensor Based Morphometry (TBM) 42 based on the analysis of the Jacobian determinant of the deformations. In this case, rather than looking for a perfect alignment of anatomical regions, we are interested in working with smooth and plausible deformations. In fact, even though a good intensity matching of images can be obtained with very spiky and unregular deformations, they can hardly be used for reliable statistical comparisons and quantifications. For instance the statistical power in TBM studies largely depends on the smoothness of the Jacobian determinant maps associated to the 
deformations. On the same line, we are interested in the plausibility of the registration in the clinical context. When applied to the longitudinal setting, a reliable non-linear registration framework should provide meaningful and robust measures of anatomical changes. The issue was explained in an exemplary way in [21, where the authors discussed a clear example of plausible non-linear registration result in Alzheimer's disease, which however led to inconsistent quantifications of volume change. The problem is intrinsically linked to the robustness of the registration, to regularity issues, and more generally to the consistency of the numerical schemes adopted by the framework, for instance for the computation of the Jacobian determinant associated to the transformation. These points are seldom discussed when presenting novel registration methods.

Finally, since non-linear registration is more and more applied to the analysis of large datasets, computational efficiency and flexibility are becoming important and desired requirements for the application in clinic and research.

In this work we present the $L C C$-demons, a reliable and general new registration framework aimed to jointly satisfy the following set of fundamental requirements: accuracy, robustness to bias, theoretical solidity coupled with numerical efficiency, and consistency of the anatomical measures. We detail in the following sections the above requirements and the related critical issues.

\subsection{Similarity Measures to Robustly Detect the Anatomical Differences}

In non-linear registration the deformation is found by optimizing a measure of similarity between two images. Thus, the quality of the retrieved deformations greatly depends on the choice of this metric. A classical similarity measure is the sum of squared differences (SSD) of the intensities, which is completely driven by the global intensity differences [6, 48]. Despite the simple numerical implementation, this metric is highly sensitive to the intensity biases which affect the medical images. Bias correction is often performed prior to image registration in order to remove global intensity inhomogeneity [45, 24, 52, but this is often not sufficient to remove local changes in the bias field. For this reason, more complex similarity criterion able to account for the bias have been proposed. For instance the (normalized) correlation criteria assumes a global affine relationship between the intensities in the images, to account for global multiplicative and additive bias [16, 14, while the (normalized) mutual information (NMI) does not require any parametric assumption on the relationship between the intensities, and is based on the global joint intensity histogram [57, 49, 35. The robustness of NMI comes at the price of the computation of the histogram, and thus of complex optimization schemes.

More importantly, all the above criteria are global, i.e. they assume a uniform bias distribution over the image space. However in medical images the bias is frequently locally varying, and in this case a global similarity measure might lead to wrong estimations of the deformations. By assuming that the information in the image is locally sufficient to estimate the intensity bias, in [11 the authors proposed a local implementation of the correlation criteria. Interestingly, such a framework led to an efficient optimization scheme based on 
Gaussian convolutions and computed through the classical Demons registration setting.

The local correlation coefficient (or local cross correlation) is based on the implicit estimation of the local affine scaling parameters of the intensities (additive plus multiplicative), and was later used in several successful registration algorithms as a good trade-off between the SSD, in which there are no hidden parameters to estimate, and the very unconstrained Mutual Information, which requires the estimation of the joint probability distribution of the intensities in the images $23,25,5$. The local formulation of LCC is indeed possible thanks to the low number of hidden parameters which enable a reliable estimation in reasonably small neighborhoods, while this is not the case for MI.

\subsection{Diffeomorphic Registration: Mathematical Formulation and Numerical Ef- ficiency}

The new-generation non-linear registration algorithms perform diffeomorphic registration by parameterizing the deformations by the flow of time varying or stationary tangent velocity fields. The use of diffeomorphisms provides a rich mathematical setting for elegant and grounded methods for atlas building [26], group-wise [8], and longitudinal statistical analysis of deformations 44, 32, 15, 17.

Diffeomorphic registration was introduced with the large deformation diffeomorphic metric mapping setting (LDDMM) 7], which parameterizes the deformations with time varying velocity fields, and in [5 an implementation of the LDDMM based on the local cross correlation criteria was proposed. However, the LDDMM has high computational cost (usually reported in order of hours on standard computers) that might prevent the intensive application on large dataset, or on high resolution data.

In order to find an optimal compromise between accuracy and computational efficiency, it was proposed in [1] to parameterize diffeomorphic transformations with stationary velocity fields (SVF). The framework was used in different registration settings [56, 8, 2, 39], and was applied to several clinical problems [50, 37, 32, 43, 38, 44, 30. The computational time reported for the registration parameterized by SVF is usually of dozens of minutes. In light of these results, the SVF registration might represent a powerful clinical instrument for the evaluation of the morphological changes in organs, due to its high flexibility and efficiency. However, standard SVF based algorithms such as the log-Demons 56 are based on the sum of squared differences criteria (SSD), which is not robust to the intensity bias affecting the medical images and might limit the applicability of such a framework in the clinical context.

\subsection{Consistent Measures of Spatial Changes from Local to Regional Scale}

In order to provide a useful measure of anatomical changes for clinically oriented applications, a registration framework should be able to consistently quantify the changes at different spatial scales.

Classically, non-linear registration was used to provide local measures of change at the finer scale (voxels, meshes) to be used in group-wise statistical 
analyses of morphological differences. Among the many techniques we can find the voxel compression maps (VCM) 20, the voxel/tensor-based morphometry based on the Jacobian determinant of the deformation (VBM, TBM) [3, 42], the RAVENS maps [41, and the cortical pattern analysis [51]. However, measures at the voxel level are sensitive to biases and are variable across subjects.

Global measures of regional changes are more commonly used in clinical practice, to quantify regional volumes on selected anatomical structures. These measures are usually quantified by segmentation: for instance, the boundary shift of anatomical regions of interest (ROI) 22] is currently used to evaluate the longitudinal volume changes in time series of images [29. As a drawback, methods based on segmentation do not allow to model the anatomical differences, for instance by voxel-wise statistical analysis or by extrapolating longitudinal observations, which is something that is possible with non-linear registration.

Global measures of average volume change can be estimated from local measures by integration of the Jacobian determinant of the deformation in selected regions [9, 12. Even though the Jacobian determinant is a largely used index of volume changes associated to anatomical deformations, it comes with some important issues that should be accounted for. First, the computation of the Jacobian matrix requires to evaluate spatial derivatives which are usually done by finite differences on the image grid, and it is well known that they are highly sensitive to the approximation introduced by the discretization. Moreover, the impact of the numerical approximations is even larger if we consider that the Jacobian determinant is basically a cubic polynomial of the values of the vector field. Second, experimental evidence showed that the logarithmic transformation of the Jacobian determinant might be more appropriate in morphometric studies, since it provides non-skewed quantities 28. As a drawback, the logJacobian determinant does not represent anymore the volume change, and thus lacks of a precise biological interpretation.

Other quantifications of spatial changes might then be considered. For instance, the flux of the deformation across the boundary of a region is a measure of global morphological change that can be used for growth and longitudinal volume change analyses [13. The flux should theoretically be less sensitive to discretization errors of the displacement field as it measures a linear polynomial of the vector across the boundaries. However, since the flux requires the computation of the vector's normal to the boundaries, the use of this measure was very limited in the past due to the high sensitivity to segmentation errors.

\subsection{Paper Organization and summary}

The present work proposes a novel symmetric diffeomorphic registration framework based on SVFs, which implements the LCC as similarity measure. We show that our registration is at the same time accurate, robust to the intensity biases and to the asymmetric image resampling. Moreover, we contribute with novel numerically stable and efficient methods to compute the Jacobian determinant and the flux of the deformation across boundaries, in order to provide consistent measures of anatomical changes from the local to the regional level. 
In Section 2 we introduce the symmetric LCC-Demons, a registration framework based on the log-Demons which implements the symmetric local Correlation Criteria (LCC) as a similarity measure. While the LCC similarity metric is well known in the literature, our contribution is to propose a new intrinsically symmetric version of it. In Section 3 we derive from the resulting SVF a spatially robust and consistent evaluation of the morphological changes from the voxel to the regional level. In fact we show that the SVF framework provides both stable voxel-by-voxel estimations of the Jacobian determinant, and consistent measures of regional changes given by the flux of the deformation across boundaries, which is obtained by the integration of the log-Jacobian determinant. Interestingly, with our framework the flux of the deformation is computed by a volume integral, which consequently leads to more robust and reliable measures of volume change. The presented method is validated in section 4.3 by comparing our algorithm against state-of-the-art registration algorithms for the accuracy in inter-subject registration, and in section 5 for the longitudinal atrophy measurements in Alzheimer's disease. The resulting longitudinal atrophy measures are compared to the ones obtained by the BSI algorithm 22, a validated measure of brain atrophy currently employed in the clinical setting.

\section{LCC-Demons: Symmetric Unbiased Diffeomorphic Registration}

\subsection{The log-Demons Algorithm}

Diffeomorphisms Parameterized by Stationary Velocity Fields (SVFs).

Let $\Omega$ be the spatial domain of a given image $F$, and let consider the manifold $\operatorname{Diff}(\Omega)$ of automorphisms of $\Omega$. The log-Demons algorithm estimates the diffeomorphic transformation $\phi \in \operatorname{Diff}(\Omega)$ which minimizes the intensity difference between a fixed image $F$ and a moving image $G$ [56]. Denote by $T_{i d} \operatorname{Diff}(\Omega)$ the tangent space of $\operatorname{Diff}(\Omega)$ at the identity. The deformation $\phi$ belongs to the one-parameter subgroup of diffeomorphisms generated by a tangent SVFs of $T \operatorname{Diff}_{i d}(\Omega)$. The one-parameter subgroup of a SVF $\mathbf{v}$ is the unique solution of:

$$
\frac{\partial \phi(x, t)}{\partial t}=\mathbf{v}(\phi(x, t)),
$$

with initial condition $\phi(x, 0)=i d$. The one-parameter subgroup $\phi_{t}(x)=\phi(x, t)$ is an additive group with respect to the real parameter $t: \phi_{s+t}(x)=\phi(x, s) \circ$ $\phi(x, t)=\phi(x, s+t)$. The transformation $\phi$ is then defined as the Lie group exponential map $\exp (\mathbf{v})=\phi(x, 1)=\phi(x)$.

The use of SVFs simplifies the LDDMM formulation and leads to a good compromise between theory and efficiency for computationally tractable registrations. For example, the exponential operation is efficiently implemented in the log-Demon's algorithm with the "scaling and squaring" scheme [1] by taking advantage of the additive property of the elements of the one-parameter subgroups. This allows to compute the final parameterization as the recursive composition of successive exponentials (Algorithm 1). 
Algorithm 1 Computing the transformation $\phi=\exp (\mathbf{v})$ parameterized by a SVF v: Scaling and Squaring for the Lie group exponential.

1. Scaling step.

Choose $N$ so that $2^{-N} \mathbf{v}$ is "small".

2. Compute a first approximation of $\phi_{0} \leftarrow \exp \left(2^{-N} \mathbf{v}\right) \approx i d+2^{-N} \mathbf{v}$

3. Squaring step.

For $k=1$ to $N$ do $\phi_{k} \leftarrow \phi_{k-1} \circ \phi_{k-1}$

Log-Demons Registration Energy.

In the log-Demons framework, the registration of the images $F$ and $G$ is achieved through the alternate minimization of the following energy, which is optimized with respect to the transformation SVF $\mathbf{v}$, and to the auxiliary correspondence field parameterized by a SVF $\mathbf{v}_{\mathbf{x}}$ [56]:

$$
\begin{aligned}
E\left(\mathbf{v}, \mathbf{v}_{\mathbf{x}}, F, G\right) & =\frac{1}{\sigma_{i}^{2}}\left\|F-G \circ \exp \left(\mathbf{v}_{\mathbf{x}}\right)\right\|_{L_{2}}^{2} \\
& +\frac{1}{\sigma_{x}^{2}}\left\|\log \left(\exp (-\mathbf{v}) \circ \exp \left(\mathbf{v}_{\mathbf{x}}\right)\right)\right\|_{L_{2}}^{2}+\frac{1}{\sigma_{T}^{2}} \operatorname{Reg}(\mathbf{v}) .
\end{aligned}
$$

Here the $L^{2}$ norm is the standard Euclidean norm, while the parameter $\sigma_{i}$ relates to the noise in the images, $\sigma_{x}$ controls the uncertainty of the matching in the coupling term, and $\sigma_{T}$ the regularization strength.

The Baker-Campbell-Hausdorff (BCH) formula describes the composition of transformations in the log-space [8]:

$$
\begin{aligned}
B C H(\mathbf{v}, \mathbf{w}) & =\log (\exp (\mathbf{v}) \circ \exp (\mathbf{w}))= \\
& =\mathbf{v}+\mathbf{w}+\frac{1}{2}[\mathbf{v}, \mathbf{w}]+\frac{1}{12}[\mathbf{w},[\mathbf{w}, \mathbf{w}]]-\frac{1}{12}[\mathbf{w},[\mathbf{v}, \mathbf{w}]]+\ldots
\end{aligned}
$$

By using the notation $\delta \mathbf{v}=B C H\left(-\mathbf{v}, \mathbf{v}_{\mathbf{x}}\right)=\log \left(\exp (-\mathbf{v}) \circ \exp \left(\mathbf{v}_{\mathbf{x}}\right)\right)$ for the so-called update field, we can rephrase the coupling term of 20 as $\|\delta \mathbf{v}\|_{L_{2}}^{2}$.

The minimization of the above energy is alternatively operated with respect to the two variables $\mathbf{v}$ and $\mathbf{v}_{\mathbf{x}}$ in two steps:

- Step1. Optimization of the Similarity. Given v, the energy

$$
E_{\text {sym }}(\mathbf{v}, \delta \mathbf{v}, F, G)=\frac{1}{\sigma_{i}^{2}}\|F-G \circ \exp (\mathbf{v}) \circ \exp (\delta \mathbf{v})\|_{L_{2}}^{2}+\frac{1}{\sigma_{x}^{2}}\|\delta \mathbf{v}\|_{L_{2}}^{2},
$$

is optimized for $\delta \mathbf{v}$, and hence for the correspondence parameter $\mathbf{v}_{\mathbf{x}}=$ $B C H(\mathbf{v}, \delta \mathbf{v})$, to find an un-regularized correspondence $\mathbf{v}_{\mathbf{x}}$ that matches the images $F$ and $G$. The Gauss-Newton optimization leads to a closed form solution for the update $\delta \mathbf{v}$, which is then efficiently composed with $\mathbf{v}$ by using the $\mathrm{BCH}$ formula. 
- Step2. Regularisation. Given $\mathbf{v}_{\mathbf{x}}$, the functional

$$
E_{\mathrm{reg}}\left(\mathbf{v}, \mathbf{v}_{\mathbf{x}}\right)=\frac{1}{\sigma_{x}^{2}}\left\|\log \left(\exp (-\mathbf{v}) \circ \exp \left(\mathbf{v}_{\mathbf{x}}\right)\right)\right\|_{L_{2}}^{2}+\frac{1}{\sigma_{T}^{2}} \operatorname{Reg}(\mathbf{v})
$$

is optimized with respect to $\mathbf{v}$. In the log-Demons the zeroth order approximation of the BCH formula $\delta \mathbf{v}=B C H\left(-\mathbf{v}, \mathbf{v}_{\mathbf{x}}\right) \simeq-\mathbf{v}+\mathbf{v}_{\mathbf{x}}$ is normally used. With this choice, by following [36] we obtain a closed form by convolution for the regularization step. When the criterion Reg is conveniently $\operatorname{chosen}^{1}$ the optimal $\mathbf{v}$ is obtained in the Fourier domain and corresponds to the Gaussian smoothing $\mathbf{v}=G_{\sigma} * \mathbf{v}_{\mathbf{x}}$, which leads to Laplacian-like regularization of the velocity field $\mathbf{v}_{\mathbf{x}}$. In addition to this, the standard log-Demons registration implements a fluid-like regularization of the update field $G_{\sigma_{f}} * \delta \mathbf{v}$, which corresponds to the choice of the projection of the update field into a smoother space of velocity fields.

\subsection{Symmetric Forces in the log-Demons}

In the log-Demons algorithm, the estimation of the SVF $\mathbf{v}$ is unbiased with respect to the choice of fixed and moving image. In fact it is symmetrically computed by minimizing the energy $E_{\text {sym }}^{\text {old }}=\|F-G \circ \exp (\mathbf{v})\|^{2}+\|F \circ \exp (-\mathbf{v})-G\|^{2}$. The symmetrization comes straightforwardly from the SVF parameterization of the deformations, and is optimized by averaging the solutions given by the two separate terms. However the strategy requires the separated optimization of both correspondence terms, and might be computationally costly in case of similarity terms more complex than the standard sum of squared differences implemented in the log-Demons.

In this paper we propose to symmetrize a given criteria by optimizing in the half-way space, reached by resampling both fixed and moving images in a single energy term. This way the symmetric deformation can be simultaneously computed with a single optimization procedure. This can be easily formulated within the SVF framework by considering $F \circ \exp \left(-\frac{\mathbf{v}}{2}\right)$ and $G \circ \exp \left(\frac{\mathbf{v}}{2}\right)$.

For instance, let $D^{s y m}(\mathbf{v}, F, G)=F \circ \exp \left(-\frac{\mathbf{v}}{2}\right)-G \circ \exp \left(\frac{\mathbf{v}}{2}\right)$ be the symmetric difference of intensities for a transformation parameterized by the SVF $\mathbf{v}$. Then the square of this residual image is the symmetric sum of squared difference $(\mathrm{SSD})$ energy $E_{\text {sym }}^{S S D}(\mathbf{v}, F, G)=\left\|F \circ \exp \left(-\frac{\mathbf{v}}{2}\right)-G \circ \exp \left(\frac{\mathbf{v}}{2}\right)\right\|^{2}$.

We show in Appendix A that in this case the optimization of the proposed symmetric SSD is consistent with the symmetric optimization used in the standard log-Demons algorithm.

${ }^{1}$ For instance by choosing the infinite order Tikhonov regularizer proposed in [10:

$$
\operatorname{Reg}(\mathbf{v})=\int \sum_{\Omega} \sum_{k=1}^{\infty} \alpha_{k}\left(\sum_{i_{1}+\ldots+i_{k}=k}\left\|\partial_{i_{1}} \ldots \partial_{i_{k}} \mathbf{v}\right\|^{2} /\left(\sigma_{d}^{2 k} k !\right)\right)
$$




\subsection{Symmetric LCC in the log-Demons}

In the standard log-Demons algorithm the correspondence field is given by the minimization of the sum of squared difference (SSD) between the intensities of the two images, which is not robust to the local intensity biases. In order to avoid mistaking spurious intensity variations for morphological differences, we propose to adapt the log-Demons framework to the local correlation coefficient (LCC), by following [11.

Consider the image $F$, and let $\bar{F}=\mathbf{G}_{\sigma} * F(x)$ be the local mean image defined by Gaussian smoothing $\mathbf{G}_{\sigma}$ with kernel size $\sigma$. The LCC is defined as:

$$
\rho(F, G)=\int_{\Omega} \frac{\overline{F G}}{\sqrt{\overline{F^{2}} \overline{G^{2}}}},
$$

The LCC similarity $\rho$ varies between -1 and 1 and measures how the intensities of the two images are correlated within the local Gaussian neighborhood of size $\sigma$.

Given a pair of images $F^{\prime}$ and $G^{\prime}$, let us consider the symmetric resampling $F=F^{\prime} \circ \exp \left(-\frac{\mathbf{v}}{2}\right)$ and $G=G^{\prime} \circ \exp \left(\frac{\mathbf{v}}{2}\right)$, and denote

$$
\rho\left(\mathbf{v}, F^{\prime}, G^{\prime}\right)=\rho(F, G)=\frac{\overline{F^{\prime} \circ \exp \left(-\frac{\mathbf{v}}{2}\right) G^{\prime} \circ \exp \left(\frac{\mathbf{v}}{2}\right)}}{\sqrt{\left[F^{\prime} \circ \exp \left(-\frac{\mathbf{v}}{2}\right)\right]^{2}} \overline{\left[G^{\prime} \circ \exp \left(\frac{\mathbf{v}}{2}\right)\right]^{2}}} .
$$

If we replace the SSD in Eq. (2) and (3) by the squared LCC, we obtain the new correspondence energy

$$
\begin{aligned}
E_{\text {sym }}^{L C C}\left(\mathbf{v}, \delta \mathbf{v}, F^{\prime}, G^{\prime}\right) & =-\frac{1}{\sigma_{i}^{2}} \rho^{2}\left(B C H(\mathbf{v}, \delta \mathbf{v}), F^{\prime}, G^{\prime}\right)+\frac{1}{\sigma_{x}^{2}}\|\delta \mathbf{v}\|_{L_{2}}^{2}= \\
& =-\frac{1}{\sigma_{i}^{2}} \rho^{2}(\delta \mathbf{v}, F, G)+\frac{1}{\sigma_{x}^{2}}\|\delta \mathbf{v}\|_{L_{2}}^{2} .
\end{aligned}
$$

We show in Appendix B that the optimization of (5) with respect to the symmetric update by $\exp \left(\frac{\partial \mathbf{v}}{2}\right)$ of $F$ and $G$ can be computed with a closed form formula:

$$
\delta \mathbf{v}=-\frac{2 \Lambda}{\|\Lambda\|^{2}-\frac{4}{\rho^{2}} \frac{\sigma_{i}^{2}}{\sigma_{x}^{2}}},
$$

where

$$
\Lambda=\left(\frac{\mathbf{G}_{\sigma} *\left(F \nabla G^{T}\right)}{\mathbf{G}_{\sigma} *(F G)}-\frac{\mathbf{G}_{\sigma} *\left(G \nabla F^{T}\right)}{\mathbf{G}_{\sigma} *(F G)}+\frac{\mathbf{G}_{\sigma} *\left(F \nabla F^{T}\right)}{\mathbf{G}_{\sigma} *\left(F^{2}\right)}-\frac{\mathbf{G}_{\sigma} *\left(G \nabla G^{T}\right)}{\mathbf{G}_{\sigma} *\left(G^{2}\right)}\right) .
$$

Thus, our symmetric LCC criterion preserves the structure of the original logDemons.

Now that we have derived an efficient registration algorithm which is robust to intensity biases, let us turn to the robust measure of longitudinal changes for the resulting deformation parameters. 


\section{Stable and Consistent Measures of Brain Changes: from Voxel to Regional Level}

The quantification of the amount of warping applied at each voxel by the dense deformation field $\phi$ is usually locally derived from the Jacobian matrix $\nabla \phi$ of the deformation in terms of determinant $\operatorname{det}(\nabla \phi)$, log-determinant $\log (\operatorname{det}(\nabla \phi))$, trace $\operatorname{Tr}(\nabla \phi)$, and the strain tensor $\nabla \phi \nabla \phi^{T}$. A global index of change can be extracted from the local information by:

- Integration of the Jacobian determinant on the region of interest $R$. This is an average measure of volume change.

- Evaluation of the flux of the deformation field across the boundaries $\partial R$ of the region, i.e. the amount of vectors flowing through the boundaries during the registration procedure. This value represents the mean shift of the boundaries, i.e. how much do we move (in the mean) along the normal to the surface enclosing the region. This is the equivalent of the classical intensity based Boundary Shift Integral 22, in which the variation of the intensities, or the "flow", along the normal to the surface is considered.

If the flux of a specific region is known, we can derive the ratio of volume change by comparing the volume enclosed by the shifted boundaries relative to the original one. However, the direct computation of the flux of a deformation is usually highly sensitive to the segmentation of the boundaries. This limitation prevented the use of the vector flux in favour of the more robust Jacobian determinant integration, while surrogate measures of the boundary shift were proposed based on the comparison of regional segmentations [22, 47].

\subsection{Flux Across Surfaces From the log-Jacobian Integration}

In this section we provide a robust method for computing the flux of the deformation within the log-Demons framework. By considering formula (1), we show in Appendix C that the following equality holds:

$$
\iiint_{R} \log (\operatorname{det}(\nabla \phi(x))) d x=\int_{0}^{1} \operatorname{flux}_{\partial R}\left(\left.\mathbf{v}\right|_{\phi(x, h)}\right) d h
$$

Equation 6, specifies that the integral of the log-Jacobian determinant of the deformation over a region $R$ is equal to the flux of the velocity field across the corresponding boundaries $\partial R$, consistently computed along the exponential trajectory. Formula (6) consistently computes the flow of the vector field during the evolution described by the SVF parameterization, and measures the flux of a vector field through the region boundaries (right side of (6) ) by scalar integration of the log-Jacobian determinant in the region volume (left side of (6p).

The present framework replaces the surface integral with the more stable volume integration, which simplifies and robustifies the measure of the flux by attenuating the segmentation errors (and relative erroneous boundary detection). This allows to deal with anatomical uncertainties, for instance by scalar 
integration on probabilistic masks. The difference between the Jacobian and the log-Jacobian analysis becomes clear: the former quantifies the mean volume changes of a region (or of a voxel as limit case), while the latter quantifies the local mean shift of the boundaries of that region (or voxel).

\subsection{A Stable Numerical Scheme for Computing the Jacobian Determinant}

The computation of the Jacobian determinant $\operatorname{det}(\nabla \phi)$ of a transformation $\phi$ is usually performed by spatial differentiation of the transformation using finite differences (Algorithm 2).

Algorithm 2 Classical computation of the Jacobian determinant by finite differences.

Given a discrete sampling $\phi$ of the transformation over the

image grid space $\left\{x_{i}\right\}$ :

1. Compute the Jacobian matrix $J^{\phi}$ via finite differences. For instance, with the forward scheme, the $k, l$ entry is:

$\nabla \phi_{k, l}(x)=\frac{\phi_{l}\left(x+h \mathbf{e}^{k}\right)-\phi_{l}(x)}{h}$, where $h$ is the scalar step size, $\phi_{l}(x)$ is the $l$-th component of the transformation, and $\mathbf{e}^{k}$ is the basis vector along the direction $k$.

2. Compute the determinant of $\nabla \phi$ with the preferred numerical method.

However, finite differences are usually highly sensitive to the spatial noise. They also critically depend on the discrete sampling which might create instabilities in case of large deformations, thus leading to incorrect Jacobian determinant estimation. For instance the sampling of the deformation field in the image grid space might introduce an unequal distribution of the vectors around a sink, and therefore induce negative Jacobian determinant estimations which are mis-interpreted as spurious folding effects.

In this section, we provide a stable and consistent computation of the Jacobian determinant according to the scaling and squaring method for the Lie group exponential (Section 2.1). From the additive property of the elements of the one-parameter subgroups, $\exp (\mathbf{v})=\exp (\mathbf{v} / 2) \circ \exp (\mathbf{v} / 2)$, the following relationship for the Jacobian determinant holds:

$$
\operatorname{det}(\nabla \exp (\mathbf{v}))=\operatorname{det}(\nabla \exp (\mathbf{v} / 2)) \circ \exp (\mathbf{v} / 2) \cdot \operatorname{det}(\nabla \exp (\mathbf{v} / 2)),
$$

The (log-)Jacobian determinant can then be recursively computed according to formula (7). If we reliably initialize the computation of the (log-)Jacobian determinant by finite differences on the scaled velocity field $\frac{\mathbf{v}}{2^{N}}$, we can then recursively compute it as detailed in Algorithm 3 .

In this case, finite differences are used only on a sufficiently small vector field in order to minimize the discretization errors. Then the Jacobian determinant is evaluated accordingly to the exponential path and is thus consistent with the definition of diffeomorphisms parameterized by SVFs. Moreover, the logJacobian determinant is defined in terms of the divergence of the velocity and, by 


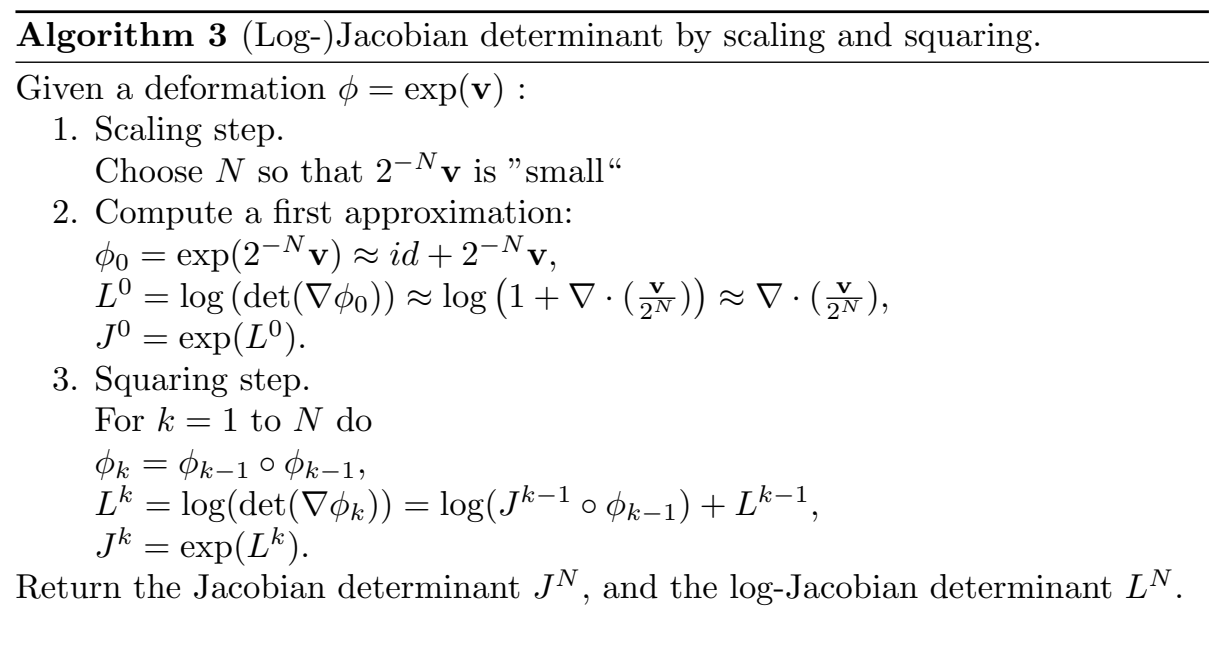

definition, the value of the corresponding Jacobian determinant always remains strictly positive.

\section{Experiments}

In this section we demonstrate the numerical stability of the methods proposed in Section 3, and the accuracy of our new LCC registration algorithm.

\subsection{Jacobian Determinant: Scaling and Squaring vs Finite Differences}

We consider here a practical example with a pair of longitudinal brain images from the ADNI dataset. As can be seen in the detail of Figure 1, even after bias correction [52] and histogram equalization, a persistent difference between the two images on the white matter intensities is still appreciable. The intensity shift in the white matter is detected by the SSD criteria of the log-Demons as an anatomical difference which generates a sink at the center of the area. This highly localized large deformation leads to negative Jacobian determinants when estimated with the standard finite differences of the sampled values on the image grid. On the contrary, our method for computing the Jacobian determinant from SVF provides stable and consistent estimations.

\subsection{Robustness to the Intensity Bias: a Controlled Example}

We first tested the robustness of the LCC-Demons to the intensity biases on a controlled experiment. We created a realistic simulated anatomical deformation based on the deformation field that matched the baseline scan $\left(I_{0}\right)$ of a patient to its 1-year follow-up of, computed using the log-Demons algorithm. The ventricular expansion was extracted for the resulting SVF $\mathbf{v}$ in a box enclosing the ventricles. The deformations in the remaining areas of the brain were imposed to be negligible random noise. The resulting deformation field 


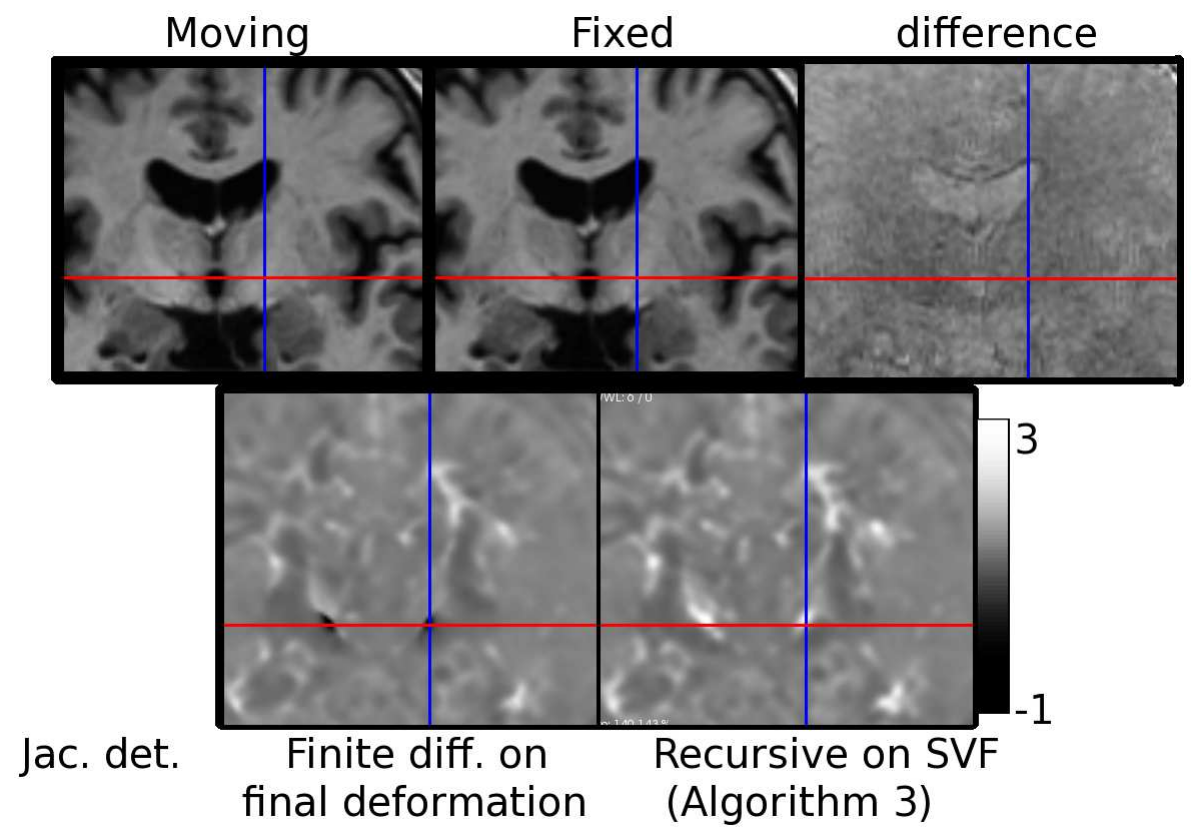

Figure 1: Stable computation of the Jacobian determinant in the SVF setting. Upper row: detail from a pair of anatomical fixed and moving brain images. The difference image denotes a mild non-stationary intensity bias detectable in the white matter. Even after bias field correction, the SSD criteria of the log-Demons models the general shift of the intensities by estimating a contracting deformation field towards the white matter. Bottom row: corresponding Jacobian determinant maps estimated by the standard finite differences on the final deformation field (left), and by the recursive scaling and squaring formula on the SVF (right). In the areas where the estimated deformation is maximal (crossing of blue and red axis) the standard finite differences lead to negative values for the Jacobian determinant.

$\varphi=\operatorname{Exp}(\mathbf{v})$ was used to warp the baseline scan $I_{0}$ to generate the longitudinal image with increased ventricular expansions. This pair of images was then used as reference to test the robustness of the detection of the longitudinal changes in the ventricular reference region to the bias.

For this purpose, the intensities of the follow-up image were corrupted by introducing spatially smooth random additive $( \pm 5 \%$ of the mean baseline intensities) and multiplicative noise (range [0.9-1.1]). The changes between baseline and generated follow-up were evaluated with the LCC-Demons and the standard $\log$-Demons as average log-Jacobian determinant values measured in the ventricles mask. The regularization parameters were set for both methods as $\sigma_{\text {fluid }}=0.5$, and $\sigma_{\text {Laplacian }}=1.5$, while the LCC smoothing parameters was 


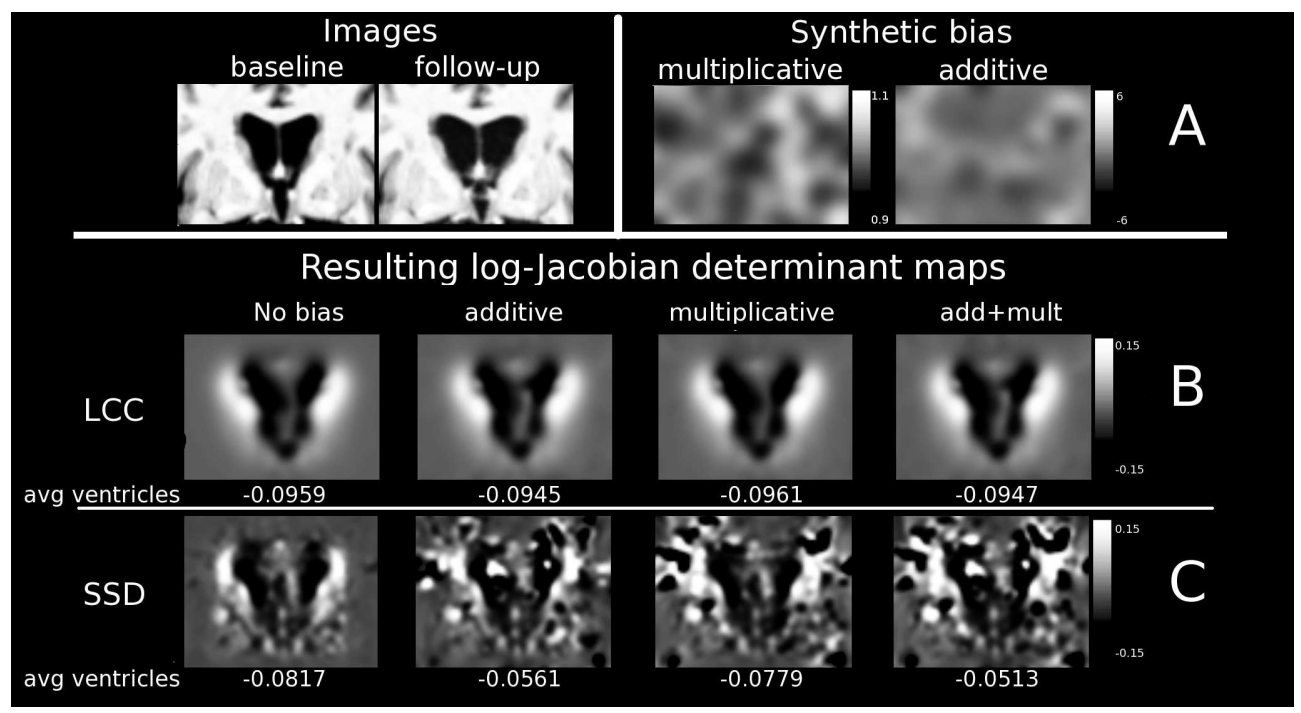

Figure 2: Synthetic experiment. A) Left: baseline and simulated follow-up ventricles expansion. Right: synthetic additive and multiplicative bias. Bottom rows: bias effect on the log-Jacobian determinant maps for the deformations estimated by the Demons algorithm with B) LCC and C) SSD similarity criteria. The LCC estimation remains consistent independently from the biases introduced.

$\sigma_{L C C}=2$. An histogram matching of the image intensities was applied prior to the standard log-Demons registration.

As can be seen in Figure 2, the LCC-Demons estimation remains stable regardless to the level of noise, while the standard SSD-based log-Demons appears to be highly sensitive. This is reflected by the regional integration of the log-Jacobian map in the ventricles mask: the SSD criteria leads to unstable evaluations while the LCC measures remain consistent.

\subsection{Registration Accuracy: Evaluation on Public Datasets}

In 27] the authors benchmarked several registration algorithms on a collection of publicly available brain images, to compare the registration performance on the matching of a set of manually labeled anatomical regions. This work represents a valuable source of information for the comparison of new registration methods, since the detailed description of the registration results is freely available ${ }^{2}$ Interestingly, the authors found that the performance of the registration algorithms was little affected by the choice of subject population, labeling protocol, and type of overlap measure.

\footnotetext{
${ }^{2}$ http://www.mindboggle.info/papers/evaluation_NeuroImage2009.php
} 
In order to test the LCC-Demons we replicated the registration pipeline proposed by [27] on the data considered by the authors (CUMC12, MGH10, LPBA40 and IBSR12 datasets). Within each dataset, we non-linearly registered all the possible pairs of linearly aligned images, after an initial affine registration to the MNI reference space [18. The registration parameters for the LCCDemons were: $\sigma_{L C C}=5, \sigma_{\text {elastic }}=1.5, \sigma_{\text {fluid }}=0.5$, and $\sigma_{i} / \sigma_{x}=0.05$, with a multi resolution scheme of 30x99x10 iterations (coarser to finer).

The registration accuracy between each source $S$ and target $T$ was evaluated by the measures of target and union overlap, defined for a specific anatomical region $r$ respectively as

$$
T O_{r}=\frac{\left|S_{r} \cap T_{r}\right|}{\left|T_{r}\right|} \text { and } M O_{r}=2 \frac{\left|S_{r} \cap T_{r}\right|}{\left|S_{r}\right|+\left|T_{r}\right|},
$$

where $|\cdot|$ is the regional volume.

In Figure 5 we can observe the performance on the LPBA40 dataset in terms of resulting mean target and union overlap on the 56 labeled regions. The results produced by the LCC-Demons compare favorably with those provided by the state-of-art algorithms, and in particular improve the ones obtained by the classical Demons registration. In the LPBA40 dataset the LCC-Demons perform significantly better than most of the compared methods, except ART, and SyN. All the reported mean differences were significant to the standard paired t-test. When tested on the other datasets (Figure 6, 7, and 8), the only algorithms that consistently provided better overlaps were again ART, and SyN. The average registration time on the tested data was of 27 minutes $( \pm 2.3)$ for a single core on a Xeon platform $2.66 \mathrm{Ghz}$ quad core, $4 \mathrm{~Gb}$ RAM.

We stress that the registration test was here performed without any specific optimization of the parameters. Moreover, even though high overlap ratios are usually indices of good registration accuracy, these values do not take into account the smoothness of the resulting transformation, nor the accuracy of the related measure of anatomical changes, e.g. of the associated Jacobian determinant. For instance, Figure (3) shows a comparison of the registration results for Syn and the LCC-Demons for a sample pairs of images of the IBSR18 dataset. Syn algorithm was applied by using the parameters specified in the paper of Klein et al ${ }^{3}$. It can be noticed that both algorithms provide reasonable results in terms of image matching. However, the Jacobian determinant map associated to the deformation estimated by Syn is more localized and looks more spiky (non-smooth). We recall that a smoother deformation potentially leads to more stable statistical analysis and often to higher statistical power in group-wise studies.

3 http://www.mindboggle.info/papers/evaluation_NeuroImage2009/ SupplementaryMaterial_Klein_NeuroImage2009.pdf 


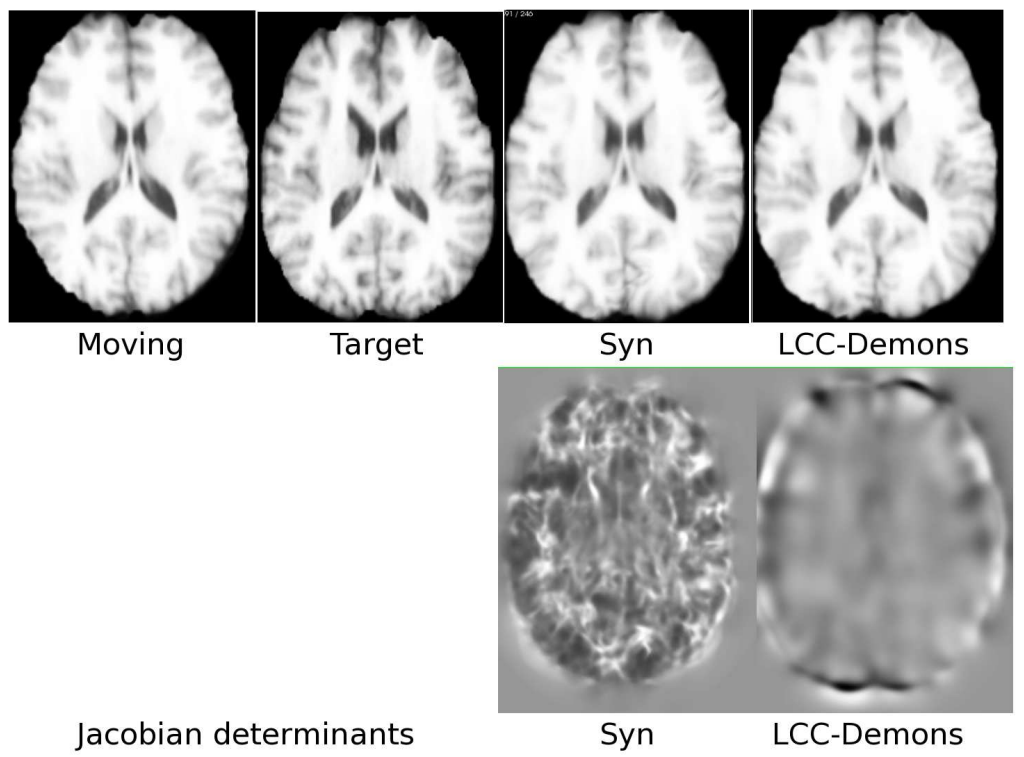

Figure 3: Example of registration result from the IBSR18 dataset. The result obtained with Syn leads to a more localized and spiky (non-smooth) deformation, while the one obtained with the LCC-Demons is smoother.

\section{Measuring the Longitudinal Changes in Alzheimer's Disease}

\subsection{Experimental Data.}

Data used in the preparation of this article were obtained from the Alzheimer's Disease Neuroimaging Initiative (ADNI) database (adni.loni.ucla.edu). The ADNI was launched in 2003 by the National Institute on Aging (NIA), the National Institute of Biomedical Imaging and Bioengineering (NIBIB), the Food and Drug Administration (FDA), private pharmaceutical companies and nonprofit organizations, as a $\$ 60$ million, 5-year public-private partnership. The Principal Investigator of this initiative is Michael W. Weiner, MD, VA Medical Center and University of California - San Francisco. ADNI is the result of efforts of many coinvestigators from a broad range of academic institutions and private corporations, and subjects were recruited from over 50 sites across the U.S. and Canada. For up-to-date information, see www.adni-info.org.

\subsection{Longitudinal Pre-processing and Registration.}

The baseline and one year follow-up brain images were collected from the ADNI dataset for a group of 200 healthy subjects and 141 patients affected by Alzheimer's disease. For each subject, the follow-up images were rigidly aligned to the baseline and the longitudinal changes were evaluated by registration 


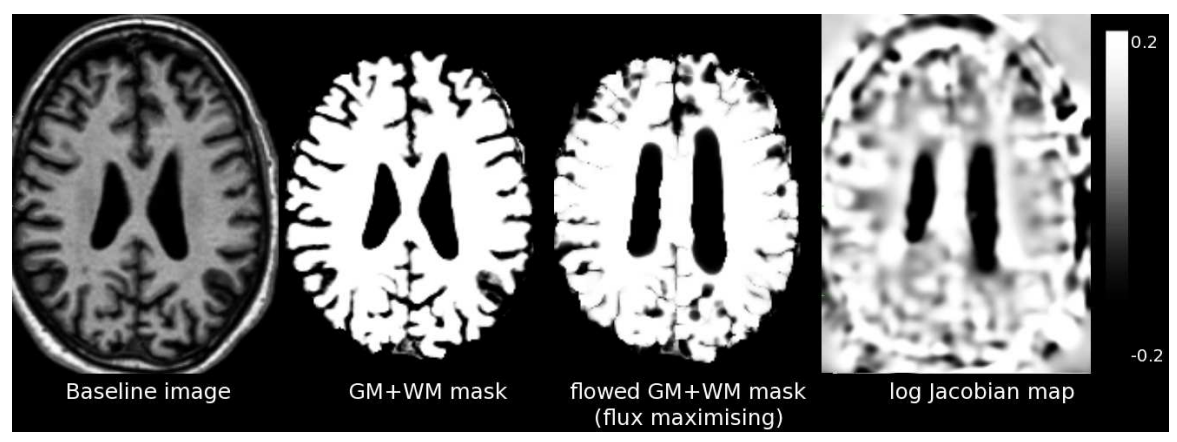

Figure 4: Deforming a volumetric mask in order to maximise the flux across the boundaries. From left: reference image, associated brain mask, and the deformed mask which maximises the flux of the longitudinal deformation. The last picture shows the log-Jacobian determinant map of deformation. It can be seen that the deformed mask is adapted to the areas of maximum expansion.

with the LCC-Demons algorithm (smoothing sigma for the criteria $\sigma_{L C C}=2$, $\sigma_{\text {elastic }}=1.5$, and $\left.\sigma_{\text {fluid }}=0.5\right)$.

\subsection{Mask Definition for Regional Measures.}

In standard deformation based morphometry, the amount of measured regional brain atrophy is usually quantified by the scalar integration of the average Jacobian determinant map of the deformation on a pre-defined region of interest (ROI).

As showed in Figure 3, morphological changes can be equally explained by different registration models. In particular, different registrations can provide very different results in terms of smoothness of the estimated deformations, which would finally lead to different localization and related quantification of the estimated volume changes.

In order to evaluate the anatomical changes consistently with respect to the registration model, we propose here to adapt a given anatomical ROI in order to maximise the vector flux across the boundaries. In this way the ROI is modified in order to account for the smoothness of the deformation. In [53] it was shown that, given a vector field $\mathbf{v}$ and a surface $S$, the maximal flux of $\mathbf{v}$ across $S$ is obtained by evolving the region along the direction

$$
\frac{\partial S}{\partial t}=(\nabla \cdot \mathbf{v}) \boldsymbol{n}
$$

Thus, given an initial brain mask and a longitudinal deformation, we can continuously deform the mask in order to maximise the flux of the longitudinal deformation through its boundaries, i.e. adapt the mask to the areas of significant longitudinal changes. 
In our experiments we computed the gray-white matter tissue mask with an automated procedure based on the FSL package tools for the automatic brain extraction and the tissue class segmentation [46, 40. The estimated mask was then flowed along the longitudinal deformation according to $(8)$ as $M^{i+1}=$ $M^{i} \circ \frac{\partial S}{\partial t}$ (15 iterations) and then used for the longitudinal quantification (Figure 4).

The whole brain changes were defined by the weighted Jacobian determinant, which represents the average volume change within the probabilistic mask, and by the weighted log-Jacobian determinant, which represents the expected flux of the deformation through the region's boundaries. If we approximate the region with a sphere S1 having the same volume, we can compute the flux-derived volume change by considering a radial field acting on the sphere S1 and having the same flux. We obtain then a volume change index by comparing the volume of the resulting shifted sphere S2 relatively to S1.

For sake of comparison the measurements were compared to the KNBSI atrophy index 29] obtained using our initial brain masks on the same processed data.

\subsection{Longitudinal Atrophy Estimation.}

The average measures for the one-year whole brain changes estimated by the LCC Demons are shown in Table 1 . The volume changes measured by the Jacobian determinant integration are consistent with those derived from the flux and are respectively of $1.8 \%$ per year for the $\mathrm{AD}$ group and $1 \%$ per year for the healthy subjects. The proposed results are consistent with the KNBSI estimations (last column), obtained on the same data. The sample size analysis provided similar results, with the lowest score given by the flux associated to the deformation (552 subjects). We specify that the KNBSI algorithm was applied here using a different processing protocol than the one proposed in [29]. In particular, the affine registration employed here was not symmetric, and there was no manual intervention in the segmentation of the brain masks. Therefore, the suboptimal processing protocol might explain the worse results in terms of sample size analysis when compared to those reported by the authors. Even though a detailed comparison of the processing procedures is out of the scope of this work, we notice that the methods performed similarly when applied to the same data.

\section{Conclusions and Perspectives}

In this work we proposed an efficient, accurate and robust registration framework for the estimation and quantification of anatomical changes in medical images. We first introduced the LCC-Demons, a diffeomorphic registration algorithm robust to intensity biases, which extends the standard log-Demons

\footnotetext{
${ }^{4} \mathrm{KNBSI}$ is available at http://sourceforge.net/projects/bsintegral/
} 


\begin{tabular}{|l|c|c|c|c|}
\hline Group & \multicolumn{3}{|c|}{ LCC-Demons } & KNBSI \\
\hline & Jacobian & Flux & Flux derived \% change & \% change \\
\hline \hline Ctrls & $1.011(0.0102)$ & $0.252(0.233)$ & $1.09(1.02)$ & $1.069(0.925)$ \\
\hline AD & $1.0186(0.011)$ & $0.409(0.239)$ & $1.81(1.06)$ & $1.714(0.989)$ \\
\hline Sample size (95\% CI) & $619(305,1154)$ & $552(309,1260)$ & $544(315,1255)$ & $590(332,1328)$ \\
\hline
\end{tabular}

Table 1: Longitudinal whole brain changes in Alzheimer's disease and healthy aging measured by the LCC-Demons as the average Jacobian determinant, flux across the boundaries, and flux derived volume change (standard deviation on parenthesis). Last column: KNBSI atrophy rates obtained on the same data. Bottom row: estimated sample size associated to the measures for detecting a $25 \%$ change in the $\mathrm{AD}$ trend when controlled to normal aging ( $80 \%$ power, $\mathrm{p}<0.05[19]$ ).

algorithm by preserving the simple numerical implementation and the related computational efficiency. Second, we provided a new numerical scheme for the computation of the Jacobian determinant of a deformation parameterized by a stationary velocity field, which prevents the numerical inaccuracies induced by the finite differences, and is consistent with the diffeomorphic parameterization. Finally, we explained the theoretical difference between log-Jacobian and Jacobian analysis of deformation fields, by showing that the surface integral of the flux of a stationary velocity field is the regional integration of log-Jacobian determinant associated to the deformations. This last contribution shows the complementary information provided by the Jacobian and the log-Jacobian determinant, and their different meaning when used as index of anatomical changes in morphometric studies.

The proposed methods were extensively tested on large publicly available dataset in both inter and intra-subject registration settings, and the results were comparable with those obtained by the most referenced methods for registration and atrophy quantification. The experiments demonstrate that LCC-Demons is a candidate instrument for both research and clinically oriented purposes, as already showed in scientific works based on the presented method [31, 34, 33.

\section{Acknowledgment}

This work was partially funded by the European Research Council (ERC advanced Grant MedYMA), ANR blanc Karametria and the EU project Care4Me.

Data collection and sharing for the experiments of Section 5 was funded by the Alzheimer's Disease Neuroimaging Initiative (ADNI) (National Institutes of Health Grant U01 AG024904). ADNI is funded by the National Institute on Aging, the National Institute of Biomedical Imaging and Bioengineering, and through generous contributions from the following: Abbott; Alzheimer's Association; Alzheimers Drug Discovery Foundation; Amorfix Life Sciences Ltd.; AstraZeneca; Bayer HealthCare; BioClinica, Inc.; Biogen Idec Inc.; BristolMyers Squibb Company; Eisai Inc.; Elan Pharmaceuticals Inc.; Eli Lilly and 
Company; F. Hoffmann-La Roche Ltd and its affiliated company Genentech, Inc.; GE Healthcare; Innogenetics, N.V.; IXICO Ltd.; Janssen Alzheimer Immunotherapy Research \& Development, LLC.; Johnson \& Johnson Pharmaceutical Research \& Development LLC.; Medpace, Inc.; Merck \& Co., Inc.; Meso Scale Diagnostics, LLC.; Novartis Pharmaceuticals Corporation; Pfizer Inc.; Servier; Synarc Inc.; and Takeda Pharmaceutical Company. The Canadian Institutes of Health Research is providing funds to support ADNI clinical sites in Canada. The grantee organization is the Northern California Institute for Research and Education, and the study is coordinated by the Alzheimer's Disease Cooperative Study at the University of California, San Diego. ADNI data are disseminated by the Laboratory for Neuro Imaging at the University of California, Los Angeles. This research was also supported by NIH grants P30 AG010129 and K01 AG030514.

\section{Appendix A. Symmetric Forces in the log-Demons}

In this section we show that the optimization of the symmetric SSD proposed in Section 2.2 is consistent with the optimization scheme of the symmetric logDemons algorithm [55]. For this purpose, we show that the Taylor expansion of the corresponding energies leads to the same form for the first order terms.

Let $D(\mathbf{v}, F, G)=F-G$ oxp $(\mathbf{v})$, be the difference of intensities for a transformation parameterized by the SVF $\mathbf{v}$, then the square of this image is the classical sum of squared difference (SSD) energy $E^{S S D}(\mathbf{v}, F, G)=\|F-G \circ \exp (\mathbf{v})\|_{L_{2}}^{2}=$ $\|D(\mathbf{v}, F, G)\|_{L_{2}}^{2}$.

By considering the symmetric difference $D^{s y m}(\mathbf{v}, F, G)=F \circ \exp \left(-\frac{\mathbf{v}}{2}\right)-G \circ$ $\exp \left(\frac{\mathbf{v}}{2}\right)$ this energy can be symmetrized into $E_{\text {sym }}^{S S D}(\mathbf{v}, F, G)=\left\|D^{\text {sym }}\right\|_{L_{2}}^{2}$. The gradient of $D^{\text {sym }}(\mathbf{v}, F, G)$ is

$$
\nabla D^{\text {sym }}=-\frac{1}{2}\left(\nabla\left[F \circ \exp \left(-\frac{\mathbf{v}}{2}\right)\right]+\nabla\left[G \circ \exp \left(\frac{\mathbf{v}}{2}\right)\right]\right) .
$$

We now recall the efficient second-order minimization scheme (ESM) of the standard SSD $\|D(\mathbf{v}, F, G)\|_{L_{2}}^{2}$ employed in the symmetric log-Demons algorithm [55]. This optimization method is based on the implicit symmetry constraint which assumes an exact matching of the images for an optimal update $\exp \left(\delta \mathbf{u}^{\text {opt }}\right)$ of the deformation. Indeed they imposed $G \circ \exp (\mathbf{v}) \circ \exp \left(\delta \mathbf{u}^{\text {opt }}\right)=$ $F$, or equivalently that $G \circ \exp (\mathbf{v}) \circ \exp \left(\frac{\delta \mathbf{u}^{\text {opt }}}{2}\right)=F \circ \exp \left(-\frac{\delta \mathbf{u}^{\text {opt }}}{2}\right)$.

Let $\mathbf{w}=B C H(\mathbf{v}, \delta \mathbf{u})$ be the deformation parameters after an update of the SVF $\mathbf{v}$ by $\delta \mathbf{u}$. The ESM optimization scheme proposed in [55] is based on the following Hessian-free second order Taylor expansion of $D$ :

$$
\begin{aligned}
D(\mathbf{w}, F, G)= & D(\mathbf{v}, F, G) \\
& +\frac{1}{2}(\nabla D(\mathbf{w}, F, G)+\nabla D(\mathbf{v}, F, G)) \delta \mathbf{u}+O\left(\|\delta u\|^{3}\right),
\end{aligned}
$$

in which $\nabla D(\mathbf{w}, F, G)=-\nabla[G \circ \exp (\mathbf{v}) \circ \exp (\delta \mathbf{u})]$, and $\nabla D(\mathbf{v}, F, G))=-\nabla[G \circ$ $\exp (\mathbf{v})]$. Thanks to the exact matching assumption, the authors obtain the 
simplified form $-\frac{1}{2}(\nabla F+\nabla[G \circ \exp (\mathbf{v})])$ for the first order term of $\mathrm{A} .22$. The authors showed the practical advantages of using this term for the optimization of $E^{S S D}$ in terms of improved registration accuracy, and smoothness properties of the resulting deformation. We notice that this term is related to the one that we obtained with our symmetric update rule (Equation A.1) , since they both involve the average of the gradients of $F$ and $G$. We notice however that with the ESM scheme only the image $G$ is resampled, while our scheme is symmetric.

\section{Appendix B. Optimization of the LCC-Demons Correspondence}

Given a pair of images $F^{\prime}$ and $G^{\prime}$, consider the symmetric resampling $F=$ $F^{\prime} \circ \exp \left(-\frac{\mathbf{v}}{2}\right)$, and $G=G^{\prime} \circ \exp \left(\frac{\mathbf{v}}{2}\right)$. In this section we derive the update formula for the optimization of the LCC symmetric correspondence (5).

$$
E_{\text {sym }}^{L C C}\left(\mathbf{v}, \delta \mathbf{v}, F^{\prime}, G^{\prime}\right)=-\frac{1}{\sigma_{i}^{2}} \rho^{2}(\delta \mathbf{v}, F, G)+\frac{1}{\sigma_{x}^{2}}\|\delta \mathbf{v}\|_{L_{2}}^{2} .
$$

The symmetric LCC correspondence considers the symmetric resampling of the images, and is optimized with respect to the symmetric composition by the update field $\exp \left(\frac{\delta \mathbf{v}}{\mathbf{2}}\right)$. We have the following Taylor expansion:

$$
F^{\frac{\delta \mathbf{v}}{2}}=F \circ \exp \left(-\frac{\delta \mathbf{v}}{2}\right)=F-\nabla F^{T} \cdot \frac{\delta \mathbf{v}}{2}+O\left(\|\delta \mathbf{v}\|^{2}\right),
$$

and

$$
G^{\frac{\delta \mathbf{v}}{2}}=G \circ \exp \left(\frac{\delta \mathbf{v}}{2}\right)=G+\nabla G^{T} \cdot \frac{\delta \mathbf{v}}{2}+O\left(\|\delta \mathbf{v}\|^{2}\right) .
$$

The updated LCC term is then written in the following way:

$$
\rho(\delta \mathbf{v}, F, G)=\frac{\mathbf{G}_{\sigma} *\left[F^{\frac{\delta \mathrm{v}}{2}} G^{\frac{\delta \mathrm{v}}{2}}\right]}{\left(\mathbf{G}_{\sigma} *\left(\left[F^{\frac{\delta \mathrm{v}}{2}}\right]^{2}\right)\right)^{\frac{1}{2}}\left(\mathbf{G}_{\sigma} *\left(\left[G^{\frac{\delta \mathrm{v}}{2}}\right]^{2}\right)\right)^{\frac{1}{2}}} .
$$

The updated factor at the denominator can be approximated as follows:

$$
\begin{aligned}
\left(\mathbf{G}_{\sigma} *\left(\left[F^{\frac{\delta \mathbf{v}}{2}}\right]^{2}\right)\right)^{-\frac{1}{2}} & \simeq\left(\mathbf{G}_{\sigma} *\left(F^{2}-F \nabla F^{T} \cdot \delta \mathbf{v}\right)\right)^{-\frac{1}{2}}+O\left(\|\delta \mathbf{v}\|^{2}\right) \\
& \simeq\left(\mathbf{G}_{\sigma} *\left(F^{2}\right)\right)^{-\frac{1}{2}}\left(1-\frac{\mathbf{G}_{\sigma} *\left(F \nabla F^{T} \cdot \delta \mathbf{v}\right)}{\mathbf{G}_{\sigma} *\left(F^{2}\right)}\right)^{-\frac{1}{2}}+O\left(\|\delta \mathbf{v}\|^{2}\right) \\
& \simeq\left(\mathbf{G}_{\sigma} *\left(F^{2}\right)\right)^{-\frac{1}{2}}\left(1+\frac{\mathbf{G}_{\sigma} *\left(F \nabla F^{T} \cdot \delta \mathbf{v}\right)}{2 \mathbf{G}_{\sigma} *\left(F^{2}\right)}\right)+O\left(\|\delta \mathbf{v}\|^{2}\right) \\
& \simeq \frac{1}{\sqrt{\mathbf{G}_{\sigma} *\left(F^{2}\right)}}+\frac{\mathbf{G}_{\sigma} *\left(F \nabla F^{T} \cdot \delta \mathbf{v}\right)}{2\left(\mathbf{G}_{\sigma} *\left(F^{2}\right)\right)^{\frac{3}{2}}}+O\left(\|\delta \mathbf{v}\|^{2}\right) .
\end{aligned}
$$

In the same way, we have

$$
\left(\mathbf{G}_{\sigma} *\left(\left[G^{\delta \mathbf{v}}\right]^{2}\right)\right)^{-\frac{1}{2}} \simeq \frac{1}{\sqrt{\mathbf{G}_{\sigma} *\left(G^{2}\right)}}-\frac{\mathbf{G}_{\sigma} *\left(G \nabla G^{T} \cdot \delta \mathbf{v}\right)}{2\left(\mathbf{G}_{\sigma} *\left(G^{2}\right)\right)^{\frac{3}{2}}}+O\left(\|\delta \mathbf{v}\|^{2}\right),
$$


and the expansion for the updated LCC term can thus be rewritten as

$$
\begin{aligned}
\rho(\delta \mathbf{v}, F, G) & \simeq \mathbf{G}_{\sigma} *\left(\left(F-\nabla F^{T} \frac{\delta \mathbf{v}}{2}\right)\left(G+\nabla G^{T} \frac{\delta \mathbf{v}}{2}\right)\right)\left(\mathbf{G}_{\sigma} *\left(\left[F^{\frac{\delta \mathbf{v}}{2}}\right]^{2}\right)\right)^{-\frac{1}{2}}\left(\mathbf{G}_{\sigma} *\left(\left[G^{\delta \mathbf{v}}\right]^{2}\right)\right)^{-\frac{1}{2}} \\
& +O\left(\|\delta \mathbf{v}\|^{2}\right) .
\end{aligned}
$$

By multiplying and by keeping only the first order term we obtain

$$
\begin{aligned}
\rho(\delta \mathbf{v}, F, G) & \simeq \rho(F, G)+\frac{1}{2} \frac{\mathbf{G}_{\sigma} *\left(F \nabla G^{T} \cdot \delta \mathbf{v}-G \nabla F^{T} \cdot \delta \mathbf{v}\right)}{\sqrt{\mathbf{G}_{\sigma} *\left(F^{2}\right) \mathbf{G}_{\sigma} *\left(G^{2}\right)}} \\
& +\frac{\rho}{2}\left(\frac{\mathbf{G}_{\sigma} *\left(F \nabla F^{T} \cdot \delta \mathbf{v}\right)}{\mathbf{G}_{\sigma} *\left(F^{2}\right)}-\frac{\mathbf{G}_{\sigma} *\left(G \nabla G^{T} \cdot \delta \mathbf{v}\right)}{\mathbf{G}_{\sigma} *\left(G^{2}\right)}\right)+O\left(\|\delta \mathbf{v}\|^{2}\right) .
\end{aligned}
$$

We denote $\rho(F, G)=\rho$. With the assumption of a sufficiently smooth update field such that for each image $I, \mathbf{G}_{\sigma} *\left(\nabla I^{T} \cdot \delta \mathbf{v}\right) \simeq \mathbf{G}_{\sigma} *\left(\nabla I^{T}\right) \cdot \delta \mathbf{v}$, we finally obtain:

$$
\begin{aligned}
\rho(\delta \mathbf{v}, F, G) & \simeq \rho+\frac{\rho}{2}\left(\frac{\mathbf{G}_{\sigma} *\left(F \nabla G^{T}\right)}{\mathbf{G}_{\sigma} *(F G)}-\frac{\mathbf{G}_{\sigma} *\left(G \nabla F^{T}\right)}{\mathbf{G}_{\sigma} *(F G)}\right. \\
& \left.+\frac{\mathbf{G}_{\sigma} *\left(F \nabla F^{T}\right)}{\mathbf{G}_{\sigma} *\left(F^{2}\right)}-\frac{\mathbf{G}_{\sigma} *\left(G \nabla G^{T}\right)}{\mathbf{G}_{\sigma} *\left(G^{2}\right)}\right) \delta \mathbf{v}+O\left(\|\delta \mathbf{v}\|^{2}\right) \\
& =\rho+\frac{\rho}{2} \Lambda \delta \mathbf{v}+O\left(\|\delta \mathbf{v}\|^{2}\right),
\end{aligned}
$$

with

$$
\Lambda=\left(\frac{\mathbf{G}_{\sigma} *\left(F \nabla G^{T}\right)}{\mathbf{G}_{\sigma} *(F G)}-\frac{\mathbf{G}_{\sigma} *\left(G \nabla F^{T}\right)}{\mathbf{G}_{\sigma} *(F G)}+\frac{\mathbf{G}_{\sigma} *\left(F \nabla F^{T}\right)}{\mathbf{G}_{\sigma} *\left(F^{2}\right)}-\frac{\mathbf{G}_{\sigma} *\left(G \nabla G^{T}\right)}{\mathbf{G}_{\sigma} *\left(G^{2}\right)}\right) .
$$

The approximated squared LCC is therefore

$$
\rho^{2}(\delta \mathbf{v}, F, G) \simeq\left(\rho+\frac{\rho}{2} \Lambda \delta \mathbf{v}\right)^{2}=\rho^{2}\left(1+\frac{1}{2} \Lambda \delta \mathbf{v}+\frac{1}{4} \delta \mathbf{v}^{T} \Lambda^{T} \Lambda \delta \mathbf{v}\right),
$$

whose gradient and Hessian are respectively $\nabla \rho^{2}(\delta \mathbf{v}, F, G)=\frac{\rho^{2}}{2} \Lambda$, and $H\left(\rho^{2}(\delta \mathbf{v}, F, G)\right)=$ $\frac{\rho^{2}}{4} \Lambda^{T} \Lambda$. To optimal of B.1 is then given by the equation:

$$
\left(-H\left(\rho^{2}(\delta \mathbf{v}, F, G)\right)+\frac{\sigma_{i}^{2}}{\sigma_{x}^{2}} \mathrm{Id}\right) \delta \mathbf{v}=\nabla \rho^{2}(\delta \mathbf{v}, F, G),
$$

whose solution is

$$
\delta \mathbf{v}=-\frac{2 \Lambda}{\|\Lambda\|^{2}-\frac{4}{\rho^{2}} \frac{\sigma_{i}^{2}}{\sigma_{x}^{2}}},
$$

thanks to the Sherman Morrison formula [54]. 


\section{Appendix C. Flux Across Surfaces From the Integration of the log- Jacobian Determinant}

In this section we prove equality (6). Let $\phi$ be a transformation parameterized by the stationary velocity field $\mathbf{v}$. The Jacobi's formula for the derivative of the Jacobian determinant of a function $\phi(x, t)$ states that:

$$
\frac{\partial \operatorname{det}(\nabla \phi(x, t))}{\partial t}=\operatorname{det}(\nabla \phi(x, t)) \operatorname{tr}\left(\nabla \phi(x, t)^{-1} \frac{\partial(\nabla \phi(x, t)}{\partial t}\right) .
$$

With reference to formula (1), by inverting the order of temporal and spatial derivatives within the trace and by applying the chain rule we have:

$$
\begin{aligned}
\operatorname{tr}\left(\nabla \phi(x, t)^{-1} \frac{\partial \nabla \phi(x, t)}{\partial t}\right) & =\operatorname{tr}\left(\nabla \phi(x, t)^{-1} \nabla \frac{\partial \phi(x, t)}{\partial t}\right) \\
& =\operatorname{tr}\left(\nabla \phi(x, t)^{-1} \nabla \mathbf{v}(\phi(x, t))\right) \\
& =\operatorname{tr}\left(\nabla \phi(x, t)^{-1} \nabla \mathbf{v}(\phi(x, t)) \nabla \phi(x, t)\right) \\
& =\operatorname{tr}(\nabla \mathbf{v}(x) \circ \phi(x, t))=\nabla \cdot(\mathbf{v}(\phi(x, t))) .
\end{aligned}
$$

This way, equation (C.1) writes as

$$
\frac{\partial \operatorname{det}(\nabla \phi(x, t))}{\partial t}=\operatorname{det}(\nabla \phi(x, t)) \nabla \cdot(\mathbf{v}(\phi(x, t)))
$$

Given a function $g(x, t)$, the solution of the ODE $\frac{\partial f(x, t)}{\partial t}=f(x, t) g(x, t)$ is $f(x, t)=\exp \left(\int_{0}^{t} g(x, t)\right)$ (up to a multiplicative constant). Taking the log of $f(x, t)=\operatorname{det}(\nabla \phi(x, t))$ we get:

$$
\log (\operatorname{det}(\nabla \phi(x, 1)))=\left.\int_{0}^{1} \nabla \cdot \mathbf{v}\right|_{\phi(x, h)} d h .
$$

The result states that, in the SVF framework, the log-Jacobian determinant of $\phi(x)=\phi(x, 1)$ is the integral of the divergence of the velocity field along the path described by the exponential.

Assuming now that $R$ is a region volume in the domain of the vector field $\phi(x)$, we can integrate (C.3) to obtain:

$$
\iiint_{R} \log (\operatorname{det}(\nabla \phi(x, 1))) d x=\int_{0}^{1}\left(\left.\iiint_{R} \nabla \cdot \mathbf{v}\right|_{\phi(x, h)} d x\right) d h .
$$

We recall now the Divergence (or Ostrogradsky's) theorem, which states that for a region $R$ immersed in a vector field $\mathbf{v}$ the following relationship holds:

$$
\iiint_{R}(\nabla \cdot \mathbf{v}) d x=\oint_{\partial R} \mathbf{v} \cdot \boldsymbol{n} d x=\operatorname{flux}_{\partial R}(\mathbf{v})
$$


where the second part of the equality represents the flux of the vector fields through the boundaries $\partial R$. Applying the Divergence Theorem to (C.4) finally gives:

$$
\iiint_{R} \log (\operatorname{det}(\nabla \phi(x))) d x=\int_{0}^{1} \operatorname{flux} \partial R\left(\left.\mathbf{v}\right|_{\phi(x, h)}\right) d h
$$

\section{References}

[1] V. Arsigny, O. Commowick, X. Pennec, N. Ayache, A Log-Euclidean framework for statistics on diffeomorphisms, in: Medical Image Computing and Computer-Assisted Intervention - MICCAI, Springer Verlag, 2006, pp. 924931.

[2] J. Ashburner, A fast diffeomorphic image registration algorithm, NeuroImage 38 (2007) 95-113.

[3] J. Ashburner, K. Friston, Voxel-based morphometry - the methods, NeuroImage 11 (2000) 805-821.

[4] B. Avants, C. Anderson, M. Grossman, J. Gee, Spatiotemporal normalization for longitudinal analysis of gray matter atrophy in frontotemporal dementia, in: Medical Image Computing and Computer-Assisted Intervention - MICCAI, Springer Verlag, 2007, pp. 303-310.

[5] B.B. Avants, C.L. Epstein, M. Grossman, J.C. Gee, Symmetric diffeomorphic image registration with cross-correlation: evaluating automated labeling of elderly and neurodegenerative brain., Medical Image Analysis 12 (2008) 26-41.

[6] R. Bajcsy, J.C. Gee, M. Reivich, Elastically deforming 3D Atlas to match anatomical brain images, Journal of Computer Assisted Tomography 17 (1993) 225-36.

[7] M.F. Beg, M.I. Miller, A. Trouvé, L. Younes, Computing Large Deformation Metric Mappings via Geodesic Flows of Diffeomorphisms, Int. J. Comput. Vision 61 (2005) 139-157.

[8] M. Bossa, M. Hernandez, S. Olmos, Contributions to 3D diffeomorphic atlas estimation: Application to brain images., in: Medical Image Computing and Computer-Assisted Intervention - MICCAI, Springer Verlag, 2007, pp. $667-674$.

[9] R. Boyes, D. Rueckert, P. Aljabar, J. Whitwell, J. Schott, D. Hill, N. Fox, Cerebral atrophy measurements using Jacobian integration: Comparison with the boundary shift integral, NeuroImage 32 (2006).

[10] P. Cachier, N. Ayache, Isotropic energies, filters and splines for vectorial regularization, J. of Math. Imaging and Vision 20 (2004) 251-265. 
[11] P. Cachier, E. Bardinet, D. Dormont, X. Pennec, N. Ayache, Iconic feature based nonrigid registration: The PASHA algorithm, Computer Vision and Image Understanding 89 (2003) 272-298. Special Issue on Nonrigid Registration.

[12] O. Camara, J. Schnabel, G. Ridgway, et al., Accuracy assessment of global and local atrophy measurement techniques with realistic simulated longitudinal Alzheimer's disease images, NeuroImage 42 (2008).

[13] M. Chung, K. Worsley, T. Paus, et al., A unified statistical approach to deformation-based morphometry, NeuroImage (2001).

[14] L.D. Collins, C.J. Holmes, T.M. Peters, A.C. Evans, Automatic 3-D modelbased neuroanatomical segmentation, Human Brain Mapping 3 (1995) 190208.

[15] B. Davis, P. Fletcher, E. Bullit, S. Joshi, Population shape regression from random design data, ICCV 4 (2007) 375-405.

[16] L. Dong, A.L. Boyer, An image correlation procedure for digitally reconstructed radiographs and electronic portal images, International Journal of Radiation Oncology,Biology, and Physics 33 (1995) 1053 - 1060.

[17] S. Durrleman, X. Pennec, A. Trouvé, N. Ayache, J. Braga, Comparison of the endocranial ontogenies between chimpanzees and bonobos via temporal regression and spatiotemporal registration, Journal of Human Evolution 62 (2012) $74-88$.

[18] V.S. Fonov, A.C. Evans, R.C. McKinstry, C.R. Almli, D.L. Collins, Unbiased nonlinear average age-appropriate brain templates from birth to adulthood, NeuroImage 47 (2009) S102.

[19] N. Fox, S. Cousens, R. Scahill, R. Harvey, M. Rossor, Using serial registered brain magnetic resonance imaging to measure disease progression in Alzheimer disease: power calculations and estimates of sample size to detect treatment effects, Arch Neurol 57 (2000) 339-344.

[20] N. Fox, W. Crum, R. Schaill, J. Stevens, J. Janssen, M. Rossnor, Imaging of onset and progression of Alzheimer's disease with voxel compression mapping of serial magnetic resonance images, Lancet 358 (2001) 201-205.

[21] N. Fox, G. Ridgway, J. Schott, Algorithms, atrophy and Alzheimer's disease: cautionary tales for clinical trials., Neuroimage 57 (2011) 15-8.

[22] P. FreeBorough, N. Fox, The boundary shift integral: An accurate and robust measure of cerebral volume changes from registered repeat MRI, IEEE Transaction on Medical Imaging 16 (1997).

[23] G. Hermosillo, O.D. Faugeras, Well-posedness of two nonrigid multimodal image registration methods, SIAM Journal of Applied Mathematics 64 (2004) 1550-1587. 
[24] Z. Hou, A Review on MR Image Intensity Inhomogeneity Correction, International Journal of Biomedical Imaging (2006).

[25] M.P. Jolly, C. Guetter, J. Guehring, Cardiac segmentation in MR cine data using inverse consistent deformable registration, in: ISBI, pp. 484-487.

[26] S. Joshi, B. Davis, B.M. Jomier, G.G. B, Unbiased diffeomorphic atlas construction for computational anatomy, Neuroimage 23 (2004) 151-160.

[27] A. Klein, J. Andersson, B. Ardekani, et al., Evaluation of 14 nonlinear deformation algorithms applied to human brain MRI registration, NeuroImage 46 (2009) 786-802.

[28] A. Leow, I. Yanovsky, M. Chiang, A. Lee, et al., Statistical properties of Jacobian maps and the realization of unbiased large-deformation nonlinear image registration, TMI 26 (2007) 822-832.

[29] K.K. Leung, M.J. Clarkson, J.W. Bartlett, S. Clegg, C.R. Jack, M.W. Weiner, N.C. Fox, S. Ourselin, Robust atrophy rate measurement in Alzheimer's disease using multi-site serial MRI: Tissue-specific intensity normalization and parameter selection, NeuroImage 59 (2009).

[30] H. Lombaert, L. Grady, X. Pennec, N. Ayache, F. Cheriet, Spectral demons - image registration via global spectral correspondence, in: ECCV (2), pp. $30-44$.

[31] M. Lorenzi, N. Ayache, G. Frisoni, X. Pennec, Probabilistic flux analysis of cerebral longitudinal atrophy, in: MICCAI 2012 Workshop on Novel Imaging Biomarkers for Alzheimer's Disease and Related Disorders.

[32] M. Lorenzi, N. Ayache, G.B. Frisoni, X. Pennec, Mapping the effects of $\mathrm{A} \beta_{1-42}$ levels on the longitudinal changes in healthy aging: hierarchical modeling based on stationary velocity fields, in: Medical Image Computing and Computer-Assisted Intervention - MICCAI, Springer Verlag, 2011, pp. 663-670.

[33] M. Lorenzi, N. Ayache, X. Pennec, Regional flux analysis of longitudinal atrophy in Alzheimer's disease., in: Medical Image Computing and Computer-Assisted Intervention - MICCAI, Springer Verlag, 2012.

[34] M. Lorenzi, N. Ayache, X. Pennec, G. Frisoni, Disentangling the normal aging from the pathological Alzheimer's disease progression on cross-sectional structural MR images, in: MICCAI Workshop on Novel Imaging Biomakers in Alzheimer's Disease.

[35] F. Maes, A. Collignon, D. Vandermeulen, G. Marchal, P. Suetens, Multimodality image registration by maximization of mutual information, IEEE Trans. Med. Imaging (1997) 187-198. 
[36] T. Mansi, X. Pennec, M. Sermesant, H. Delingette, N. Ayache, LogDemons revisited: Consistent regularisation and incompressibility constraint for soft tissue tracking in medical images, in: Medical Image Computing and Computer-Assisted Intervention - MICCAI, Springer Verlag, 2010, pp. 652659.

[37] T. Mansi, X. Pennec, M. Sermesant, H. Delingette, N. Ayache, iLogDemons: A Demons-based registration algorithm for tracking incompressible elastic biological tissues, International Journal of Computer Vision 92 (2011) 92-111.

[38] K. McLeod, C. Seiler, M. Sermesant, X. Pennec, A near-incompressible poly-affine motion model for cardiac function analysis, MICCAI Workshop on Statistical Atlases and Computational Models of the Heart: Mapping Structure and Function (STACOM2012) (2012).

[39] M. Modat, G. Ridgway, P. Daga, M. Cardoso, D. Hawkes, J. Ashburner, S. Ourselin, Log-euclidean free-form deformation, in: Proc. of SPIE Medical Imaging 2011, volume 7962, SPIE, 2011.

[40] B. Patenaude, S. Smith, D. Kennedy, M. Jenkinson, A Bayesian model of shape and appearance for subcortical brain, NeuroImage 56(3) (2011) 907-922.

[41] S. Resnik, A. Goldszal, C., Davatzikos, S. Golski, M. Kraut, E. Metter, R. Bryan, A. Zonderman, One year age changes in MRI brain volumes in older adults, Cerebral Cortex 10 (2000) 464-472.

[42] W.R. Riddle, R. Li, J.M. Fitzpatrick, S.C. DonLevy, B.M. Dawant, R.R. Price, Characterizing changes in MR images with color-coded Jacobians., Magn Reson Imaging 22 (2004) 769-777.

[43] C. Seiler, X. Pennec, M. Reyes, Geometry-aware multiscale image registration via OBBtree-based polyaffine log-Demons, in: Medical Image Computing and Computer-Assisted Intervention - MICCAI, Springern Verlag, 2011, pp. 631-638.

[44] V. Siless, J. Glaunés, P. Guevara, J.F. Mangin, C. Poupon, D. Bihan, B. Thirion, P. Fillard, Joint T1 and brain fiber Log-Demons registration using currents to model geometry, in: Medical Image Computing and Computer-Assisted Intervention - MICCAI, Springer Verlag, 2012, pp. 5765.

[45] J. Sled, A. Zijdenbos, A. Evans, A nonparametric method for automatic correction of intensity nonuniformity in mri data., IEEE Trans Med Imaging 17 (1998) 87-97.

[46] S. Smith, Fast robust automated brain extraction., Human Brain Mapping 17 (2002). 
[47] S. Smith, Y. Zhang, M. Jenkinson, J. Chen, P. Matthews, A. Federico, N.D. Stefano, Accurate, robust, and automated longitudinal and cross-sectional brain change analysis, NeuroImage 17 (2002).

[48] R. Stefanescu, Parallel nonlinear registration of medical images with a priori information on anatomy and pathology, Phd Thesis, Université de Nice - Sophia-Antipolis (2005).

[49] C. Studholme, D. Hill, D. Hawkes, Automated 3-D registration of MR and CT images of the head, Medical Image Analysis 1 (1996) 163-175.

[50] A. Sweet, X. Pennec, Log-domain diffeomorphic registration of diffusion tensor images, Proc. of WBIR: Workshop on Biomedical Image Registration (2010).

[51] P. Thompson, K. Ayashi, G. Zubicaray, A. Janke, S. Rose, J. Semple, D. Herman, M. Hong, S. Dittmer, D. Dodrell, A. Toga, Dynamics of gray matter loss in Alzheimer's disease, The Journal of Neuroscience 23 (2003) 994-1005.

[52] N. Tustison, B.A.P. Cook, Y. Zheng, A. Egan, P. Yushkevich, J. Gee, N4itk: Improved n3 bias correction, IEEE Tr on Medical Imaging 29 (2010).

[53] A. Vasilevskiy, K. Siddiqi, Flux maximizing geometric flows, IEEE Trans. Pattern Anal. Mach. Intell. 24 (2002) 1565-1578.

[54] T. Vercauteren, Image Registration and Mosaicing for Dynamic In Vivo Fibered Confocal Microscopy, Phd thesis, École Nationale Supérieure des Mines de Paris, 2008.

[55] T. Vercauteren, X. Pennec, A. Perchant, N. Ayache, Diffeomorphic demons: Efficient non-parametric image registration., NeuroImage 45 (2008) Supplement 1, Pages S61-S72.

[56] T. Vercauteren, X. Pennec, A. Perchant, N. Ayache, Symmetric Logdomain diffeomorphic registration: A Demons-based approach., in: Medical Image Computing and Computer-Assisted Intervention - MICCAI, Springer Verlag, 2008, pp. 754-761.

[57] W. Wells, P. Viola, H. Atsumi, S. Nakajima, R. Kikinis, Multi-modal volume registration by maximization of mutual information, Medical Image Analysis 1 (1996) 35-51. 
Target overlap for the LPBA40 dataset
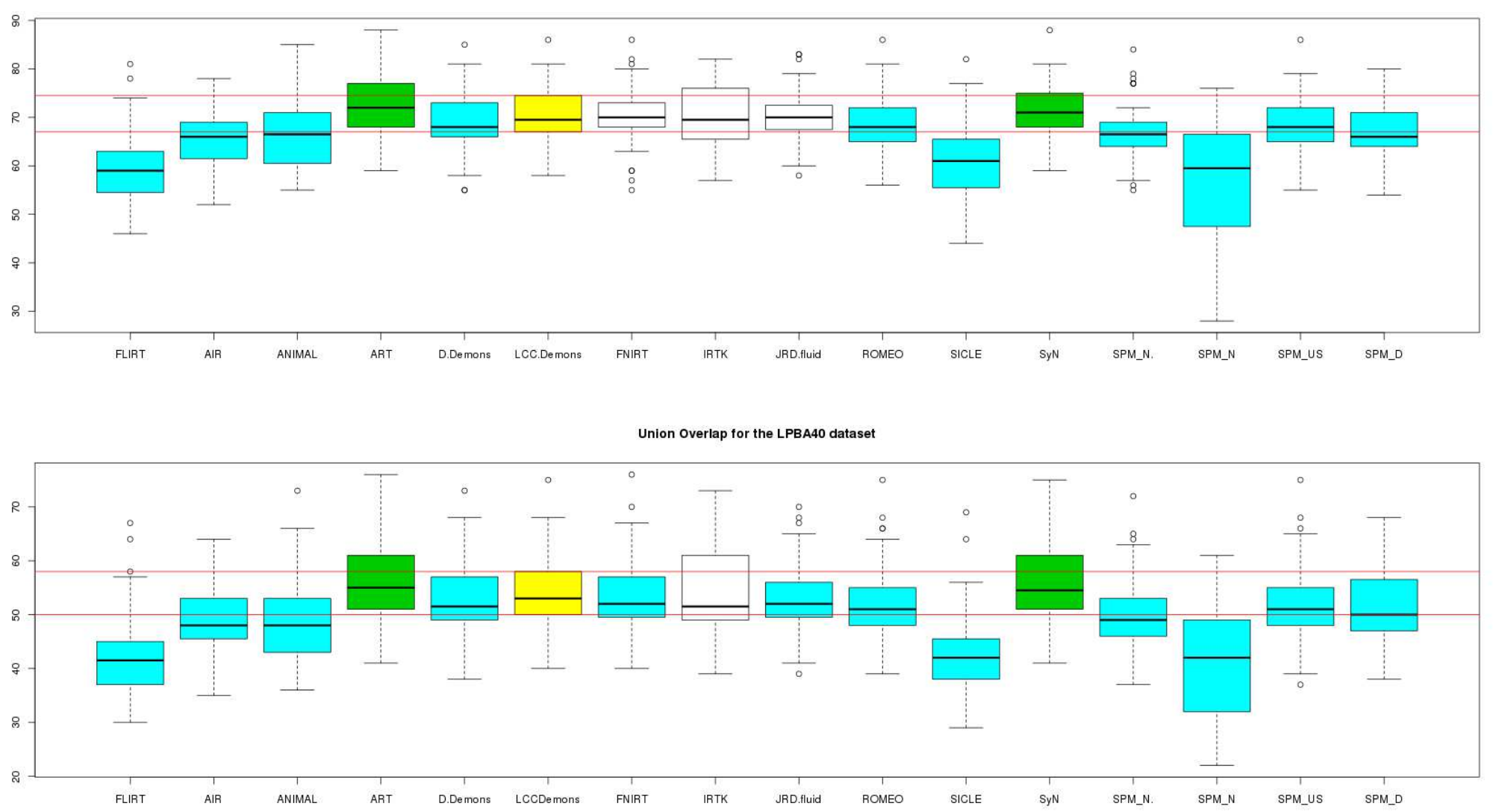

Figure 5: Inter-subject registration on the LPBA40 dataset: target and union overlap on the labeled regions. Yellow: LCCDemons. Blue: significantly lower overlaps. Green: significantly higher overlaps. White: no significant difference $(\mathrm{p}<0.05$, paired t-test on the labeled regions). Red lines: upper and lower quartiles of the LCC-Demons overlaps. 
Target Overlap for the CUMC12 dataset
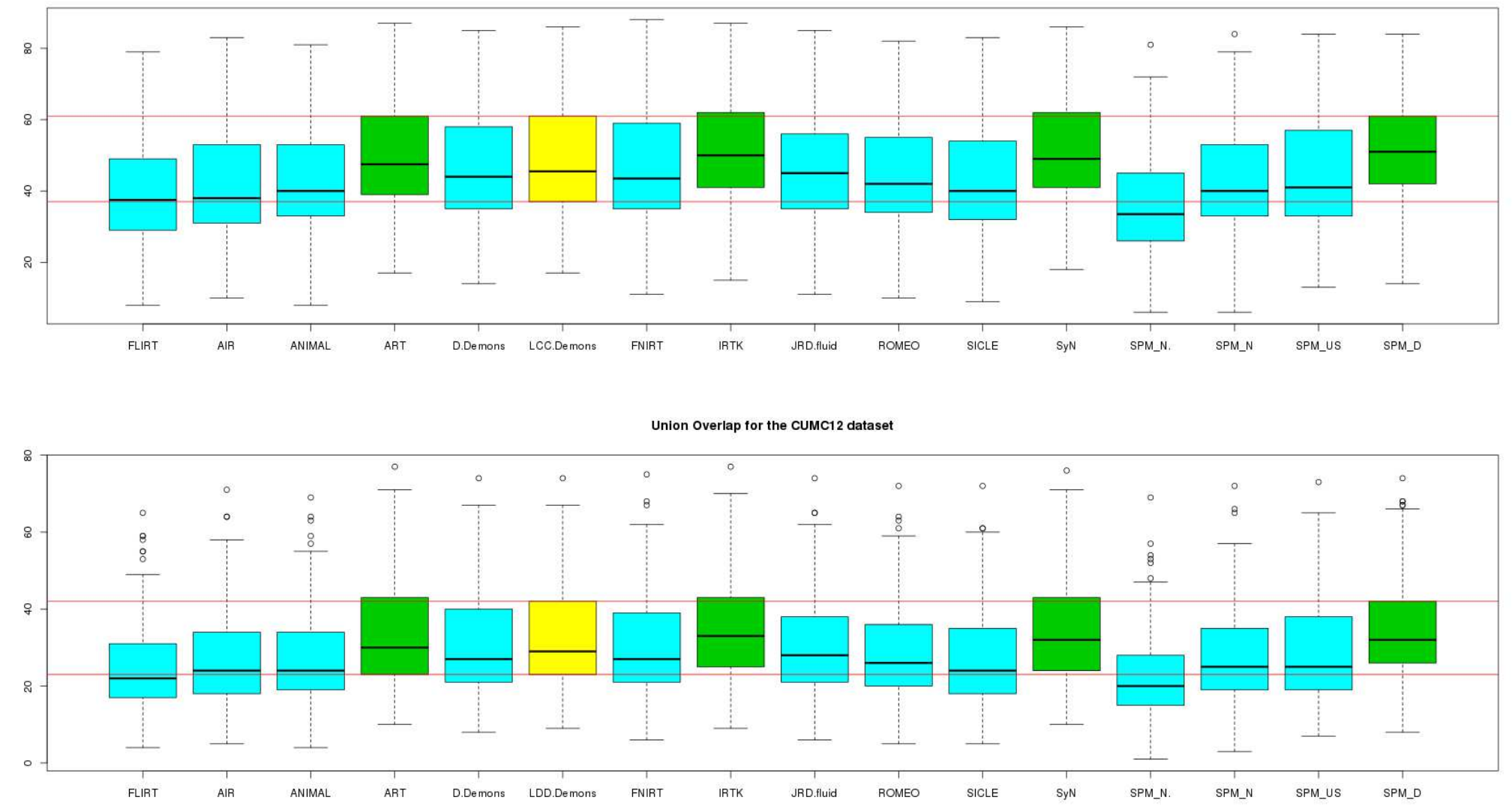

Figure 6: Inter-subject registration on the CUMC12 dataset: target and union overlap on the labeled regions. Yellow: LCCDemons. Blue: significantly lower overlaps. Green: significantly higher overlaps. White: no significant difference $(\mathrm{p}<0.05$, paired t-test on the labeled regions). Red lines: upper and lower quartiles of the LCC-Demons overlaps. 
Target Overlap for the MGH10 dataset

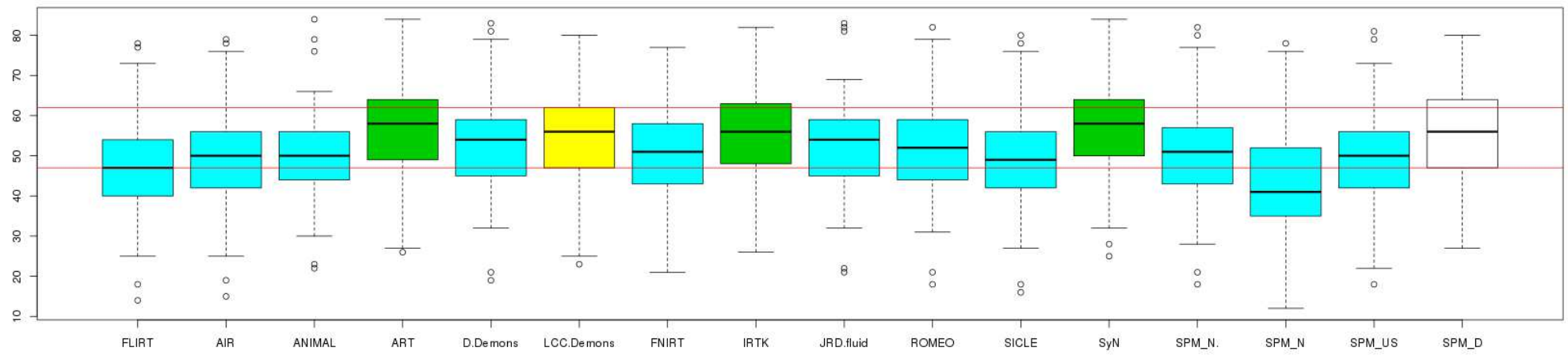

Union Overlap for the MGH10 datase

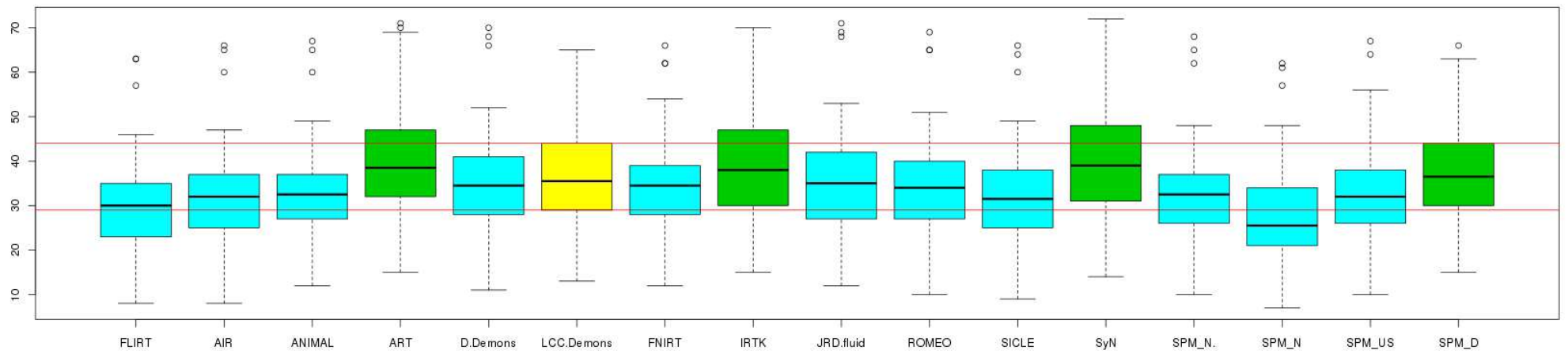

Figure 7: Inter-subject registration on the MGH10 dataset: target and union overlap on the labeled regions. Yellow: LCCDemons. Blue: significantly lower overlaps. Green: significantly higher overlaps. White: no significant difference $(\mathrm{p}<0.05$, paired t-test on the labeled regions). Red lines: upper and lower quartiles of the LCC-Demons overlaps. 
Target Overlap for the IBSR18 dataset

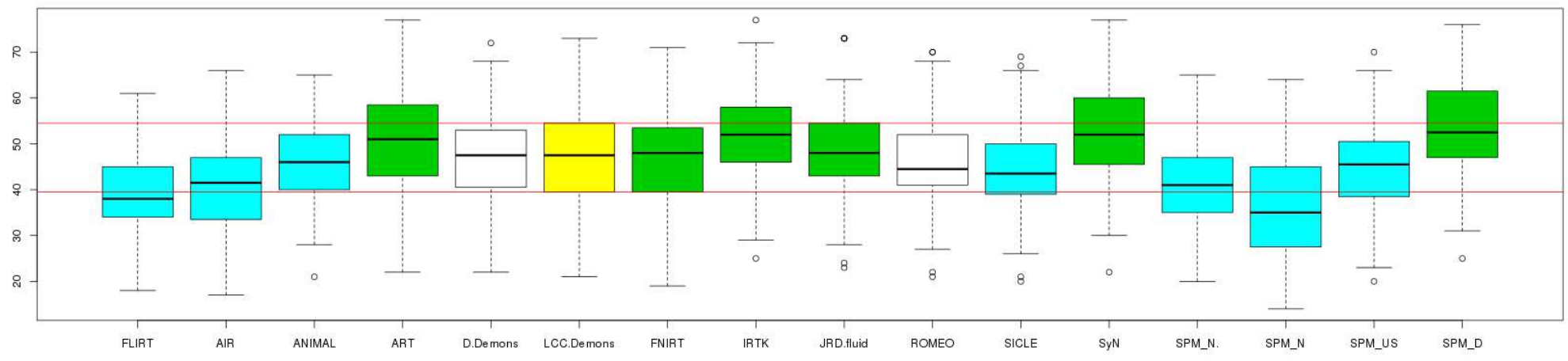

Union Overlap for the IBSR18 datase

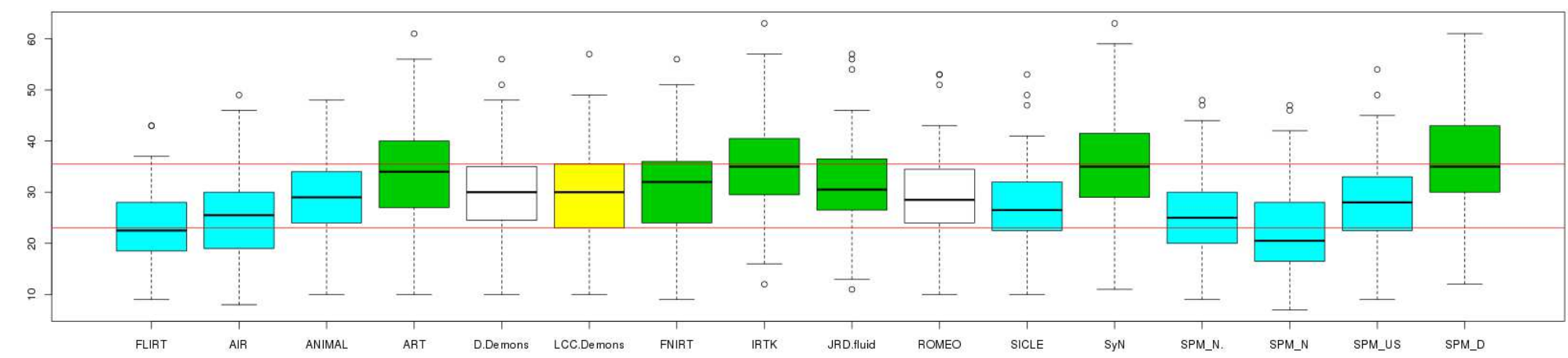

Figure 8: Inter-subject registration on the IBSR18 dataset: target and union overlap on the labeled regions. Yellow: LCCDemons. Blue: significantly lower overlaps. Green: significantly higher overlaps. White: no significant difference $(\mathrm{p}<0.05$, paired t-test on the labeled regions). Red lines: upper and lower quartiles of the LCC-Demons overlaps. 